I dent i fying pl asmonic modes in circul ar par abol oi dal geomet ry

\begin{tabular}{|l|l|}
\hline 著者 & $\begin{array}{l}\text { KURI HARA Kazuyoshi, TAKAHARA Juni chi, YAMAMOTO } \\
\text { Kazuhi ro, OTOMD Aki ra }\end{array}$ \\
\hline $\begin{array}{l}\text { j our nal or } \\
\text { publ i cat i on ti t l e }\end{array}$ & $\begin{array}{l}\text { Journal of Physi cs A. Nat hemat i cal and } \\
\text { Theor et i cal }\end{array}$ \\
\hline vol une & 42 \\
\hline number & 18 \\
\hline page r ange & $185401-1$ - 185401- 38 \\
\hline year & 2009- 05- 08 \\
\hline URL & ht t p: //hdl . handl e. net /10098/2164 \\
\hline
\end{tabular}




\section{Identifying plasmonic modes in circular paraboloidal geometry by quasi-separation of variables}

Kazuyoshi Kurihara $^{1,3}$, Junichi Takahara ${ }^{2}$, Kazuhiro Yamamoto ${ }^{1}$ and Akira Otomo $^{1}$

${ }^{1}$ Kobe Advanced ICT Research Center, National Institute of Information and Communications Technology (NICT), Kobe 651-2492, Japan

2 Department of Systems Innovation, Graduate School of Engineering Science, Osaka University, Toyonaka 560-8531, Japan

${ }^{3}$ Department of Physics, Faculty of Education and Regional Studies, University of Fukui, Fukui 910-8507, Japan

E-mail:k-kuri@nict.go.jp

Short title:

Identifying plasmonic modes in circular paraboloidal geometry 


\begin{abstract}
Analytic solutions for surface plasmon polaritons in a circular paraboloidal geometry are theoretically studied by solving the wave equation for the magnetic field in paraboloidal coordinates, using the quasi-separation of variables in combination with perturbation methods. It is found that solutions do not exist for a metallic solid paraboloid, but they can be obtained for a metallic hollow paraboloid in the form of standing waves. This paper provides the zeroth- and first-order approximate solutions of plasmonic modes for a metallic hollow paraboloid and graphically represents the zeroth-order solution in electric field-line patterns.
\end{abstract}

Keywords: surface plasmon plaritons, plasmonics, quasi-separation of variables, paraboloid, nanofocusing, superfocusing

PACS numbers: 02.30.Mv, 41.20.Jb, 52.35.Hr, 42.25.Bs, 42.25.Gy, 78.20.-e, 78.20.Bh 


\section{Contents}

1. Introduction

2. Quasi-separation of variables applied to the wave equation for a magnetic field

3. Unification of the radial equations in the two regions

4. Boundary conditions for the radial and extended angular functions

5. Application of perturbation methods for solving the extended angular equations

6. Zeroth-order approximate solutions for the metallic hollow paraboloid

6.1 Extended angular functions of the zeroth order for the metallic hollow paraboloid

6.2 Characteristic equation for determining the unified separation quantity of the zeroth order for the metallic hollow paraboloid

6.3 Approximate determination of the zeroth-order unified separation quantity based on a figure-of-merit function

6.4 A unified radial function of the zeroth order for the metallic hollow paraboloid

7. Non-existence of zeroth-order solutions for the metallic solid paraboloid

8. First-order perturbation solutions for the metallic hollow paraboloid

8.1 Extended angular functions of the first order for the metallic hollow paraboloid

8.2 Determination of the unified separation quantity of the first order for the metallic hollow paraboloid

8.3 Unified radial function of the first order for the metallic hollow paraboloid

9. Electric field-line representation

10. Conclusions

Acknowledgements

Appendix A. Differential equations for the azimuthal magnetic field of transverse magnetic waves in a parabolic system of coordinates

Appendix B. Some properties of Coulomb wave functions

Appendix C. Approximate behaviour of $\zeta_{u}^{(0)}(\xi)$ in (110) for the limit of $\xi \rightarrow \infty$

References 


\section{Introduction}

Electromagnetic waves excited at a metal-dielectric interface are known as surface plasmon polaritons (SPPs) [1]. Because of the capability of SPPs to localize and guide optical energy in nanoscale metal-dielectric structures, renewed interest in SPPs has been focused on the context of surface plasmon-based circuits [2-5], or "plasmonics," which is considered to be a strong candidate for developing the intermediary technology of merging photonics and electronics at nanoscale dimensions [5]. The fundamental building blocks in such plasmonic optoelectronic circuits are subwavelength plasmonic waveguides [6-10], which were first explored by J. Takahara (one of the authors) and his co-workers in 1997 [10]. In recent years, particular attention has been paid to tapered metal-dielectric structures such as metallic cones [11-14] and wedges [15-19] for the development of tapered plasmonic waveguides capable of delivering and concentrating optical radiation energy at the nanoscale, namely, nanofocusing the optical energy [12]. Although tapered plasmonic waveguides have been examined analytically [20-27], numerically [27-34], and experimentally [35-37], we do not have sufficient data on plasmonic modes for development of tapered plasmonic waveguides, as expected for the actual designs, such as the effects of the tip radius and the taper angle, mainly because until recently, we did not have a suitable method to solve such data in theoretical physics.

In conventional theoretical physics, the problem of plasmonic modes for the tapered plasmonic waveguides was tackled by using two analytical methods based upon the Fourier method of separation of variables (SOV) or the classical SOV (CSOV): the geometrical-optics (GO) approximation (also called the adiabatic approximation) [12, 13, 16-18, 23-27] and the local SOV (LSOV) approximation [11, 15, 20-22]. The former technique is the geometrical replacement of tapered structures by an infinite series of infinitesimal untapered structures; it is easy to determine plasmonic modes for the untapered structures by means of CSOV. The latter technique is employed to solve the wave equation for plasmonic modes by means of CSOV locally at the apex point of the tapered structures. The two analytical methods are rough approximations because an uncountably infinite number of boundary conditions at all points on the tapered surface cannot be taken into account; as a result, the tip radius effect of tapered plasmonic waveguides, which is an important factor for actual experiments, cannot be taken into 
account by using the two analytical methods based upon the CSOV. Recently, we proposed a new analytical method called the quasi-separation of variables (QSOV) [14, 19], which is employed to determine plasmonic modes for tapered plasmonic waveguides. The new analytical method is an incomplete SOV and is considered to be an extension of the CSOV. In our previous papers [14, 19], we applied this new method to metallic cones [14] and wedges [19] and successfully obtained their plasmonic modes more accurately than ever because boundary conditions at all points on the tapered surface were satisfied. Many tapered metal-dielectric structures still remain to be investigated by using the QSOV method for tapered plasmonic modes. Thus, we can attain knowledge of plasmonic modes for tapered metal-dielectric structures that would be impossibly difficult to investigate by using the previous analytical methods based on the CSOV.

In this paper, we describe a theoretical investigation of plasmonic modes in circular paraboloidal geometry by solving the wave equation for the magnetic field by means of the QSOV in combination with perturbation methods. The circular paraboloidal geometry is one of the geometries with a finite radius of curvature at the tip, which is difficult or impossible to investigate by employing the previous analytical methods based upon the CSOV. We observed that plasmonic modes cannot exist for the metallic solid paraboloid but can be obtained for the metallic hollow paraboloid as standing waves; this fact indicates that the circular paraboloidal geometry cannot be investigated by using the previous analytical methods in which only the nanofocusing (also called superfocusing ${ }^{4}$ ) can be treated approximately. Therefore, it is important to point out that the metallic solid paraboloid is a nonideal typical structure that can be used for nanofocusing, as described in [38, 39]. From the viewpoint of nanofocusing, the plasmonic modes for circular paraboloidal geometry are completely different from those for conical [14] and wedge-shaped [19] geometries; the data on the plasmonic modes could not be obtained without the new analytical method using QSOV. In order to theoretically clarify the actual nanofocusing of SPPs, which has already been discussed numerically [40, 41] and experimentally [42], the results of our theoretical study presented in this paper strongly suggest that the effects of the tip radius and the taper angle on the nanofocusing should be analytically examined in other geometries such as half two-sheet hyperboloidal geometry [43, 44] (this geometry will be studied by means of QSOV in detail elsewhere). 
${ }^{4}$ The term "superfocusing" is more general than "nanofocusing" because the former can be used for waves of any nature, whereas the latter is specific to the optical range in electromagnetic waves. 


\section{Quasi-separation of variables applied to the wave equation for a magnetic field}

We consider two circular paraboloidal structures: (1) a metallic circular paraboloid surrounded by a dielectric and (2) a dielectric circular paraboloid surrounded by metal; in this paper, the abovementioned structures are simply called metallic solid paraboloid and metallic hollow paraboloid, respectively. As shown in figure 1, the paraboloidal coordinate $(\xi, \eta, \phi)$ can be used to describe an infinite circular paraboloid with a surface $\eta=\eta_{0}$, permittivity $\varepsilon_{1}$, and surrounded by matter with permittivity $\varepsilon_{2}$. The paraboloidal coordinates are related to the rectangular coordinates $(x, y, z)$ according to the following transformations [45-47]:

$$
x=s \sqrt{\xi \eta} \cos \phi, \quad y=s \sqrt{\xi \eta} \sin \phi, \quad z=\frac{s}{2}(\xi-\eta),
$$

where $0 \leq \xi<\infty, 0 \leq \eta<\infty, 0 \leq \phi<2 \pi$ and $\mathrm{s}>0$ is a scale factor. Curves of constant $\xi$ and $\eta$ in the cross-section of the $y-z$ plane are shown in figure 2, where the $y$-and $z$-coordinates are normalized by the scale factor $s$. When the imaginary parts of the permittivities are ignored, we obtain the conditions $\varepsilon_{1}<0$ and $\varepsilon_{2}>0$ for the metallic solid paraboloid and the conditions $\varepsilon_{1}>0$ and $\varepsilon_{2}<0$ for the metallic hollow paraboloid. We consider the plasmonic modes induced by transverse magnetic (TM) waves, whose magnetic field $\boldsymbol{H}(\boldsymbol{x}, \mathrm{t})$ at time $t$ at the point located by the coordinate vector $\boldsymbol{x}$ possesses only an azimuthal component of the form

$$
\boldsymbol{H}(\boldsymbol{x}, \mathrm{t})=\left(0,0, H_{\phi}(\boldsymbol{x}, \mathrm{t})\right)
$$

in the paraboloidal system of coordinates. Assuming the harmonic time dependence $\mathrm{e}^{-\mathrm{i} \omega \mathrm{t}}$, we describe $H_{\phi}(\boldsymbol{x}, \mathrm{t})$ as

$$
H_{\phi}(\boldsymbol{x}, \mathrm{t})=\operatorname{Re}\left[H_{\phi}(\boldsymbol{x}) \mathrm{e}^{-\mathrm{i} \omega \mathrm{t}}\right],
$$

where $\omega$ is the angular frequency of interest. Substituting the $\boldsymbol{H}(\boldsymbol{x}, \mathrm{t})$ given by (2) and (3) into the vector differential equation obtained by combining the two Maxwell curl equations in the absence of sources (see Appendix A), we observe that $H_{\phi}(\boldsymbol{x})$ in (3) has an azimuthal symmetry described by

$$
H_{\phi}(\boldsymbol{x})=H_{\phi}(\xi, \eta)=\left\{\begin{array}{lc}
H_{\phi 1}(\xi, \eta), & 0 \leq \eta \leq \eta_{0} \\
H_{\phi 2}(\xi, \eta), & \eta_{0} \leq \eta<\infty,
\end{array}\right.
$$

which satisfies the following wave equation: 


$$
\frac{4 \sqrt{\xi}}{\xi+\eta} \frac{\partial^{2}}{\partial \xi^{2}}\left[H_{\phi j}(\xi, \eta) \sqrt{\xi}\right]+\frac{4 \sqrt{\eta}}{\xi+\eta} \frac{\partial^{2}}{\partial \eta^{2}}\left[H_{\phi j}(\xi, \eta) \sqrt{\eta}\right]+\varepsilon_{j} \frac{\omega^{2}}{c^{2}} H_{\phi j}(\xi, \eta)=0, j=1,2
$$

where $c$ is the velocity of light in vacuum. Equation (5) can be described more explicitly as

$$
\begin{aligned}
\frac{\partial}{\partial \xi}\left(\xi \frac{\partial}{\partial \xi} H_{\phi j}(\xi, \eta)\right)+\frac{\partial}{\partial \eta}\left(\eta \frac{\partial}{\partial \eta} H_{\phi j}(\xi, \eta)\right) \\
-\frac{1}{4}\left(\frac{1}{\xi}+\frac{1}{\eta}\right) H_{\phi j}(\xi, \eta)+\frac{\xi+\eta}{4} \varepsilon_{j} s^{2} k_{0}^{2} H_{\phi j}(\xi, \eta)=0, \quad j=1,2,
\end{aligned}
$$

where $k_{0}$ is the wave number in vacuum defined as

$$
k_{0}=\frac{\omega}{c}
$$

As described in our previous papers [14, 19], we aim to determine solutions of the following form by using the QSOV:

$$
H_{\phi j}(\xi, \eta)=\Xi_{j}(\xi) \mathrm{H}_{j}(\eta, \xi), \quad j=1,2
$$

with the boundary conditions

$$
\mathrm{H}_{j}\left(\eta_{0}, \xi\right)=1, \quad j=1,2
$$

to determine $\mathrm{H}_{j}(\eta, \xi)$ uniquely. By substituting (8) into (6), we obtain

$$
\begin{aligned}
& -\left[\frac{1}{\Xi_{j}(\xi)} \frac{\partial}{\partial \xi}\left(\xi \frac{\partial}{\partial \xi} \Xi_{j}(\xi)\right)+\left(\frac{\varepsilon_{j} s^{2} k_{0}^{2}}{4} \xi-\frac{1}{4 \xi}\right)\right]= \\
& \left\{\begin{array}{l}
\frac{1}{\mathrm{H}_{j}(\eta, \xi)} \frac{\partial}{\partial \eta}\left(\eta \frac{\partial}{\partial \eta} \mathrm{H}_{j}(\eta, \xi)\right)+\left(\frac{\varepsilon_{j} s^{2} k_{0}^{2}}{4} \eta-\frac{1}{4 \eta}\right) \\
+\frac{1}{\mathrm{H}_{j}(\eta, \xi) \Xi_{j}(\xi)}\left[\frac{\partial}{\partial \xi}\left(\xi \Xi_{j}(\xi) \frac{\partial}{\partial \xi} \mathrm{H}_{j}(\eta, \xi)\right)+\left(\frac{\partial}{\partial \xi} \mathrm{H}_{j}(\eta, \xi)\right) \xi \frac{\partial}{\partial \xi} \Xi_{j}(\xi)\right]
\end{array}\right\}, \quad j=1,2 .
\end{aligned}
$$

Because the left-hand side of (10) depends on $\xi$ alone and the right-hand side depends on both $\xi$ and $\eta$, both the sides must depend upon $\xi$ alone. By setting both sides equal to $\zeta_{j}(\xi)$, we obtain

$$
\frac{1}{\Xi_{j}(\xi)} \frac{\partial}{\partial \xi}\left(\xi \frac{\partial}{\partial \xi} \Xi_{j}(\xi)\right)+\left(\frac{\varepsilon_{j} s^{2} k_{0}^{2}}{4} \xi-\frac{1}{4 \xi}\right)=-\zeta_{j}(\xi), \quad j=1,2
$$

and 


$$
\begin{aligned}
& \frac{1}{\mathrm{H}_{j}(\eta, \xi)} \frac{\partial}{\partial \eta}\left(\eta \frac{\partial}{\partial \eta} \mathrm{H}_{j}(\eta, \xi)\right)+\left(\frac{\varepsilon_{j} s^{2} k_{0}^{2}}{4} \eta-\frac{1}{4 \eta}\right) \\
& +\frac{1}{\mathrm{H}_{j}(\eta, \xi) \Xi_{j}(\xi)}\left[\frac{\partial}{\partial \xi}\left(\xi \Xi_{j}(\xi) \frac{\partial}{\partial \xi} \mathrm{H}_{j}(\eta, \xi)\right)+\left(\frac{\partial}{\partial \xi} \mathrm{H}_{j}(\eta, \xi)\right) \xi \frac{\partial}{\partial \xi} \Xi_{j}(\xi)\right]=\zeta_{j}(\xi), \quad j=1,2 .
\end{aligned}
$$

Here, $\zeta_{j}(\xi)$ for $j=1,2$ are called quasi-separation invariants or separation quantities in analogy with the separation constants in the CSOV. By multiplying (11) by $\Xi_{j}(\xi) / \xi$ and rearranging the terms, we obtain the following radial equations:

$$
\frac{\partial^{2}}{\partial \xi^{2}} \Xi_{j}(\xi)+\frac{1}{\xi} \frac{\partial}{\partial \xi} \Xi_{j}(\xi)+\left(\frac{\varepsilon_{j} s^{2} k_{0}^{2}}{4}+\frac{\zeta_{j}(\xi)}{\xi}-\frac{1}{4 \xi^{2}}\right) \Xi_{j}(\xi)=0, \quad j=1,2 .
$$

By multiplying $(12)$ by $\mathrm{H}_{j}(\eta, \xi)$ and rearranging the terms, we obtain the following extended angular equations:

$$
\frac{\partial}{\partial \eta}\left(\eta \frac{\partial}{\partial \eta} \mathrm{H}_{j}(\eta, \xi)\right)+\left(\frac{\varepsilon_{j} s^{2} k_{0}^{2}}{4} \eta-\frac{1}{4 \eta}-\zeta_{j}(\xi)\right) \mathrm{H}_{j}(\eta, \xi)=-F_{j}(\eta, \xi), \quad j=1,2
$$

where

$$
F_{j}(\eta, \xi)=\frac{\partial^{2}}{\partial \xi^{2}} \mathrm{H}_{j}(\eta, \xi)+\left(1+\frac{2 \xi}{\Xi_{j}(\xi)} \frac{\partial}{\partial \xi} \Xi_{j}(\xi)\right) \frac{\partial}{\partial \xi} \mathrm{H}_{j}(\eta, \xi), \quad j=1,2 .
$$

We can replace (6) with (13) and (14), which satisfy the boundary conditions of (9). 


\section{Unification of the radial equations in the two regions}

The radial equations (13) are separately expressed in the two different regions $j=1,2$. Remarkably, the equations can be transformed into a unified form that is independent of the regions by considering the limiting cases $\xi \rightarrow 0+$ and $\xi \rightarrow \infty$.

For $\xi \rightarrow 0+$, because the conditions

$$
\left|\frac{\zeta_{j}(0)}{\xi}-\frac{1}{4 \xi^{2}}\right| \gg \frac{\left|\varepsilon_{j}\right| s^{2} k_{0}^{2}}{4}, \quad j=1,2 \quad(\xi \rightarrow 0+)
$$

are acceptable, (13) can be described as

$$
\frac{\partial^{2}}{\partial \xi^{2}} \Xi_{j}(\xi)+\frac{1}{\xi} \frac{\partial}{\partial \xi} \Xi_{j}(\xi)+\left(\frac{\zeta_{j}(0)}{\xi}-\frac{1}{4 \xi^{2}}\right) \Xi_{j}(\xi)=0, \quad j=1,2 \quad(\xi \rightarrow 0+),
$$

in which the material terms $\varepsilon_{j}$ are neglected, and therefore the different expressions in the regions $j=1,2$ are a superfluity of equations. By setting

$$
\begin{gathered}
\zeta_{u}(0)=\zeta_{j}(0), \quad j=1,2 \\
\Xi_{u}(\xi)=\Xi_{j}(\xi), \quad j=1,2 \quad(\xi \rightarrow 0+),
\end{gathered}
$$

we describe (17) as

$$
\frac{\partial^{2}}{\partial \xi^{2}} \Xi_{u}(\xi)+\frac{1}{\xi} \frac{\partial}{\partial \xi} \Xi_{u}(\xi)+\left(\frac{\zeta_{u}(0)}{\xi}-\frac{1}{4 \xi^{2}}\right) \Xi_{u}(\xi)=0 \quad(\xi \rightarrow 0+)
$$

in the unified notation for the two different regions. Equation (20) is used for investigating asymptotic solutions for $\Xi_{u}(\xi)$, as $\xi \rightarrow 0+$. Two nontrivial linearly independent particular solutions to (20) are expressed by

$$
\Xi_{u}(\xi)=H_{1}^{(1)}\left(2 \nu \xi^{1 / 2}\right), \quad H_{1}^{(2)}\left(2 \nu \xi^{1 / 2}\right) \quad(\xi \rightarrow 0+)
$$

with the definition

$$
v=\sqrt{\zeta_{u}(0)}
$$

where $H_{1}^{(1)}$ and $H_{1}^{(2)}$ denoted Hankel functions of the first and second kinds [45], respectively. By taking into account the time dependence $\mathrm{e}^{-\mathrm{i} \omega \mathrm{t}}$ in (3), we observe that $H_{1}^{(1)}\left(2 \nu \xi^{1 / 2}\right)$ and $H_{1}^{(2)}\left(2 \nu \xi^{1 / 2}\right)$ in (21) correspond to the outgoing and incoming waves, respectively, for $\xi \rightarrow \infty$ (using this condition may appear strange for discussions on the condition $\xi \rightarrow 0+$, but is very useful for characterization, as shown in Section 4) when $v$ in (22) represents a positive real number: 


$$
v>0
$$

It is important to point out that $H_{1}^{(1)}\left(2 \nu \xi^{1 / 2}\right)$ and $H_{1}^{(2)}\left(2 \nu \xi^{1 / 2}\right)$ in (21) do not belong to a class of functions that characterize the superfocusing (or nanofocusing) property.

For $\xi \rightarrow \infty$, because the physical situation is considered to be similar to that for SPPs in planar geometry, (13) can be described with a unified notation for the two regions, as follows:

$$
\Xi_{u}(\xi)=\Xi_{j}(\xi), \quad j=1,2 \quad(\xi \rightarrow \infty),
$$

and this unified radial function satisfies the Sommerfeld radiation conditions [47]:

$$
\lim _{r \rightarrow \infty} r\left(\frac{\partial \Xi_{u}}{\partial r} \pm i k_{p} \Xi_{u}\right)=0
$$

where

$$
r=\sqrt{x^{2}+y^{2}+z^{2}}
$$

and $k_{p}$ denotes the wave number of SPPs in the planar geometry, expressed by [48, 49]:

$$
k_{p}=\frac{\omega}{\mathrm{c}} \sqrt{\frac{\varepsilon_{1} \varepsilon_{2}}{\varepsilon_{1}+\varepsilon_{2}}} .
$$

By using (1), (25) can be transformed into the form:

$$
\lim _{\xi \rightarrow \infty} \frac{\xi+\eta}{2}\left(\frac{2}{s} \frac{\partial}{\partial \xi}+\frac{2}{s} \frac{\partial}{\partial \eta} \pm i k_{p}\right) \Xi_{u}(\xi)=0
$$

or

$$
\lim _{\xi \rightarrow \infty} \frac{\xi+\eta}{2}\left(\frac{2}{s} \frac{\partial}{\partial \xi} \pm i k_{p}\right) \Xi_{u}(\xi)=0 .
$$

This equation allows us to select simple candidates for the unified radial function, as follows:

$$
\Xi_{u}(\xi)=\exp \left(i s k_{p} \xi / 2\right), \quad \exp \left(-i s k_{p} \xi / 2\right) \quad(\xi \rightarrow \infty)
$$

which, unfortunately, do not belong to a class of solutions for (13). By carefully selecting candidates for (29) so that

$$
\Xi_{u}(\xi)=\frac{1}{\sqrt{\xi}} \exp \left(i s k_{p} \xi / 2\right), \quad \frac{1}{\sqrt{\xi}} \exp \left(-i s k_{p} \xi / 2\right) \quad(\xi \rightarrow \infty),
$$

we obtain their differential equation 


$$
\frac{\partial^{2}}{\partial \xi^{2}} \Xi_{u}(\xi)+\frac{1}{\xi} \frac{\partial}{\partial \xi} \Xi_{u}(\xi)+\left(\frac{s^{2} k_{p}^{2}}{4}-\frac{1}{4 \xi^{2}}\right) \Xi_{u}(\xi)=0 \quad(\xi \rightarrow \infty),
$$

which can be derived from (13) by setting:

$$
\zeta_{j}(\xi)=\frac{s^{2} \beta_{j}^{2}}{4} \xi \quad(\xi \rightarrow \infty)
$$

with

$$
\beta_{j}=\sqrt{k_{p}^{2}-\varepsilon_{j} k_{0}^{2}}
$$

and by using (24). Equation (32) is regarded as the limiting equation of (13) for $\xi \rightarrow \infty$.

At this stage, we are ready to look for a unified form of (13) by following an inversion process of finding the limiting equations (20) and (32) as $\xi \rightarrow 0+$ and $\xi \rightarrow \infty$, respectively. A simple candidate for the unified radial equation is expressed by

$$
\frac{\partial^{2}}{\partial \xi^{2}} \Xi_{u}(\xi)+\frac{1}{\xi} \frac{\partial}{\partial \xi} \Xi_{u}(\xi)+\left(\frac{s^{2} k_{p}^{2}}{4}+\frac{\zeta_{u}(0)}{\xi}-\frac{1}{4 \xi^{2}}\right) \Xi_{u}(\xi)=0 \quad(0 \leq \xi<\infty),
$$

which approaches (20) and (32) as $\xi \rightarrow 0+$ and $\xi \rightarrow \infty$, respectively. Then, it can be inferred without difficulty that the more general candidate is expressed by

$$
\frac{\partial^{2}}{\partial \xi^{2}} \Xi_{u}(\xi)+\frac{1}{\xi} \frac{\partial}{\partial \xi} \Xi_{u}(\xi)+\left(\frac{s^{2} k_{p}^{2}}{4}+\frac{\zeta_{u}(0)+A(\xi)}{\xi}-\frac{1}{4 \xi^{2}}\right) \Xi_{u}(\xi)=0 \quad(0 \leq \xi<\infty)(36)
$$

where $\mathrm{A}(\xi)$ is arbitrary only if

$$
\lim _{\xi \rightarrow 0} A(\xi)=0, \quad \lim _{\xi \rightarrow \infty} A(\xi) / \xi=0 .
$$

By setting

$$
A(\xi)=\zeta_{u}(\xi)-\zeta_{u}(0)
$$

we can rewrite (36) as

$$
\frac{\partial^{2}}{\partial \xi^{2}} \Xi_{u}(\xi)+\frac{1}{\xi} \frac{\partial}{\partial \xi} \Xi_{u}(\xi)+\left(\frac{s^{2} k_{p}^{2}}{4}+\frac{\zeta_{u}(\xi)}{\xi}-\frac{1}{4 \xi^{2}}\right) \Xi_{u}(\xi)=0 \quad(0 \leq \xi<\infty),
$$

where $\zeta_{u}(\xi)$ satisfies the condition

$$
\lim _{\xi \rightarrow \infty} \zeta_{u}(\xi) / \xi=0
$$

from (37) and (38). By comparing (39) with (13), we find that the unification conditions are given by 


$$
\zeta_{j}(\xi)=\zeta_{u}(\xi)+\frac{s^{2} \beta_{j}^{2}}{4} \xi, \quad j=1,2 \quad(0 \leq \xi<\infty),
$$

which allows for a unified notation of the radial functions, as follows:

$$
\Xi_{u}(\xi)=\Xi_{j}(\xi), \quad j=1,2 \quad(0 \leq \xi<\infty) .
$$

Further discussion on the unified radial equation (39) would require more detailed information on the unified separation quantity $\zeta_{u}(\xi)$, which can be determined from the boundary conditions. 


\section{Boundary conditions for the radial and extended angular functions}

In the preceding section, we showed that the original radial equations (13) are simplified to the unified radial equation (39) in which the unified radial function for $\xi \rightarrow 0+$ can be expressed by a linear combination of the outgoing wave, $H_{1}^{(1)}\left(2 \nu \xi^{1 / 2}\right)$,

and the incoming wave, $H_{1}^{(2)}\left(2 \nu \xi^{1 / 2}\right)$, as shown in (21). In this case, the unified radial function $\Xi_{u}(\xi)$ is divided into an incoming part, $\Xi_{i n}(\xi)$, and an outgoing part, $\Xi_{\text {out }}(\xi)$, as follows:

$$
\Xi_{u}(\xi)=\Xi_{\text {in }}(\xi)+\Xi_{\text {out }}(\xi),
$$

which satisfies the boundary condition

$$
\lim _{\xi \rightarrow 0+} \Xi_{\text {in }}(\xi)=\lim _{\xi \rightarrow 0+} \Xi_{\text {out }}(\xi)
$$

By using the asymptotic formulas [45]

$$
H_{1}^{(1)}\left(2 \nu \xi^{1 / 2}\right) \sim-\frac{i}{\pi \nu \xi^{1 / 2}}, \quad H_{1}^{(2)}\left(2 \nu \xi^{1 / 2}\right) \sim \frac{i}{\pi \nu \xi^{1 / 2}} \quad(\xi \rightarrow 0+)
$$

we can select from (44) the following values:

$$
\Xi_{\text {in }}(\xi) \sim-i H_{0} H_{1}^{(2)}\left(2 \nu \xi^{1 / 2}\right), \quad \Xi_{\text {out }}(\xi) \sim i H_{0} H_{1}^{(1)}\left(2 \nu \xi^{1 / 2}\right) \quad(\xi \rightarrow 0+)
$$

where $H_{0}$ denotes the complex amplitude of the magnetic field to be determined by the initial conditions. By substituting (46) into (43), we determine the boundary conditions of the unified radial function, as follows:

$$
\Xi_{u}(\xi) \sim \frac{2 H_{0}}{\pi \nu \xi^{1 / 2}} \quad(\xi \rightarrow 0+),
$$

which has an undetermined constant $v$, defined by (22), and therefore (47) is unsuitable for the practical applications. For (47), by setting

$$
H_{0}^{\prime}=\frac{H_{0}}{\nu},
$$

we obtain the practical boundary condition of the unified radial function as follows:

$$
\Xi_{u}(\xi) \sim \frac{2 H_{0}^{\prime}}{\pi \xi^{1 / 2}} \quad(\xi \rightarrow 0+) .
$$

It must be noted that this boundary condition is not limited to the case $v>0$ in (23), or 
$\zeta_{u}(0)>0$ from (22); however, it can be applied to any values of $\zeta_{u}(0) \in(-\infty, \infty)$ because the outgoing and incoming waves for the unified radial equation (39) are described by using Coulomb wave functions, as shown in (140) and (141).

In order to obtain the boundary conditions of the angular functions, the continuity of the radial electric field at the metal-dielectric surface $\left(\eta=\eta_{0}\right)$ can be employed. The Ampére-Maxwell equation in the absence of current density allows us to describe the electric field as a function of the magnetic field. Because the magnetic field possesses only the azimuthal component with azimuthal symmetry, the electric field $\boldsymbol{E}(\boldsymbol{x}, \mathrm{t})$ can be described as

$$
\boldsymbol{E}(\boldsymbol{x}, \mathrm{t})=\left(E_{\xi}(\xi, \eta, \mathrm{t}), E_{\eta}(\xi, \eta, \mathrm{t}), 0\right)
$$

in which we see that

$$
\begin{aligned}
& E_{\xi}(\xi, \eta, \mathrm{t})=\operatorname{Re}\left[E_{\xi}(\xi, \eta) \mathrm{e}^{-\mathrm{i} \omega \mathrm{t}}\right] \\
& E_{\eta}(\xi, \eta, \mathrm{t})=\operatorname{Re}\left[E_{\eta}(\xi, \eta) \mathrm{e}^{-\mathrm{i} \omega \mathrm{t}}\right]
\end{aligned}
$$

where

$$
\begin{gathered}
E_{\xi}(\xi, \eta)= \begin{cases}E_{\xi 1}(\xi, \eta), & 0 \leq \eta \leq \eta_{0} \\
E_{\xi 2}(\xi, \eta), & \eta_{0} \leq \eta<\infty\end{cases} \\
E_{\eta}(\xi, \eta)= \begin{cases}E_{\eta 1}(\xi, \eta), & 0 \leq \eta \leq \eta_{0} \\
E_{\eta 2}(\xi, \eta), & \eta_{0} \leq \eta<\infty .\end{cases}
\end{gathered}
$$

Each component of the electric field is described as

$$
\begin{array}{cc}
E_{\xi j}(\xi, \eta)=\frac{2 \mathrm{i}}{\varepsilon_{j} s k_{0} \sqrt{\xi+\eta}} \frac{\partial}{\partial \eta}\left(H_{\phi j}(\xi, \eta) \sqrt{\eta}\right), & j=1,2 \\
E_{\eta j}(\xi, \eta)=-\frac{2 \mathrm{i}}{\varepsilon_{j} s k_{0} \sqrt{\xi+\eta}} \frac{\partial}{\partial \xi}\left(H_{\phi j}(\xi, \eta) \sqrt{\xi}\right), \quad j=1,2 .
\end{array}
$$

By substituting (8) into (55), and using (42), we can rewrite the radial electric field as

$$
E_{\xi j}(\xi, \eta)=\frac{2 \mathrm{i}}{\varepsilon_{j} s k_{0} \sqrt{\xi+\eta}} \Xi_{u}(\xi) \frac{\partial}{\partial \eta}\left(\mathrm{H}_{j}(\eta, \xi) \sqrt{\eta}\right), \quad j=1,2,
$$

which is continuous at the metal-dielectric surface $\left(\eta=\eta_{0}\right)$. Subsequently, we obtain

$$
\frac{1}{\varepsilon_{1}} \lim _{\eta \rightarrow \eta_{0}-0} \frac{\partial}{\partial \eta}\left[\mathrm{H}_{1}(\eta, \xi) \sqrt{\eta}\right]=\frac{1}{\varepsilon_{2}} \lim _{\eta \rightarrow \eta_{0}+0} \frac{\partial}{\partial \eta}\left[\mathrm{H}_{2}(\eta, \xi) \sqrt{\eta}\right]
$$

for the boundary conditions of the angular functions. 


\section{Application of perturbation methods for solving the extended angular equations}

According to (41) and (42), the extended angular equations (14) and (15) can be simplified into the following expressions:

$\frac{\partial}{\partial \eta}\left(\eta \frac{\partial}{\partial \eta} \mathrm{H}_{j}(\eta, \xi)\right)+\left(\frac{\varepsilon_{j} s^{2} k_{0}^{2}}{4} \eta-\frac{1}{4 \eta}-\zeta_{u}(\xi)-\frac{s^{2} \beta_{j}^{2}}{4} \xi\right) \mathrm{H}_{j}(\eta, \xi)=-F_{j}(\eta, \xi), j=1,2$

with

$$
F_{j}(\eta, \xi)=\frac{\partial^{2}}{\partial \xi^{2}} \mathrm{H}_{j}(\eta, \xi)+\left(1+\frac{2 \xi}{\Xi_{u}(\xi)} \frac{\partial}{\partial \xi} \Xi_{u}(\xi)\right) \frac{\partial}{\partial \xi} \mathrm{H}_{j}(\eta, \xi), \quad j=1,2 .
$$

In spite of this simplification, we still face difficulty with regard to the fact that (59) and (60) include the as-yet-to-be-determined unified radial function $\Xi_{u}(\xi)$. Moreover, we continue to face difficulty in solving such a complicated partial differential equation (PDE) containing first- and second-order partial derivatives with respect to $\xi$ and $\eta$. In order to overcome such difficulties, let us apply perturbation methods to the extended angular equation (59) by placing $F_{j}(\eta, \xi)$ on the right-hand side as a perturbing term because an exact solution can be obtained at the left-hand side. According to the perturbation theory [50], we introduce the perturbation parameter $0 \leq \delta \leq 1$ into (59) and consider the perturbed equation

$$
\frac{\partial}{\partial \eta}\left(\eta \frac{\partial}{\partial \eta} \mathrm{H}_{j}(\eta, \xi)\right)+\left(\frac{\varepsilon_{j} s^{2} k_{0}^{2}}{4} \eta-\frac{1}{4 \eta}-\zeta_{u}(\xi)-\frac{s^{2} \beta_{j}^{2}}{4} \xi\right) \mathrm{H}_{j}(\eta, \xi)=-\delta F_{j}(\eta, \xi), \quad j=1,2
$$

which should have a solution of the form

$$
\mathrm{H}_{j}(\eta, \xi)=\mathrm{H}_{j}^{(0)}(\eta, \xi)+\delta \mathrm{H}_{j}^{(1)}(\eta, \xi)+\delta^{2} \mathrm{H}_{j}^{(2)}(\eta, \xi)+\cdots, \quad j=1,2 .
$$

Accordingly, $\zeta_{u}(\xi)$ and $\Xi_{u}(\xi)$ should be described as

$$
\zeta_{u}(\xi)=\zeta_{u}^{(0)}(\xi)+\delta \zeta_{u}^{(1)}(\xi)+\delta^{2} \zeta_{u}^{(2)}(\xi)+\cdots
$$

and

$$
\Xi_{u}(\xi)=\Xi_{u}^{(0)}(\xi)+\delta \Xi_{u}^{(1)}(\xi)+\delta^{2} \Xi_{u}^{(2)}(\xi)+\cdots
$$

respectively. For $\Xi_{u}(\xi)$, the incoming $\Xi_{\text {in }}(\xi)$ and the outgoing $\Xi_{\text {out }}(\xi)$ should be also described as

$$
\Xi_{i n}(\xi)=\Xi_{i n}^{(0)}(\xi)+\delta \Xi_{i n}^{(1)}(\xi)+\delta^{2} \Xi_{i n}^{(2)}(\xi)+\cdots
$$

and

$$
\Xi_{\text {out }}(\xi)=\Xi_{\text {out }}^{(0)}(\xi)+\delta \Xi_{\text {out }}^{(1)}(\xi)+\delta^{2} \Xi_{\text {out }}^{(2)}(\xi)+\cdots,
$$

respectively. By substituting (62)-(64) into (61), and setting the coefficients of the 
powers of $\delta$ equal to each other, we obtain the following system of equations for $\mathrm{H}_{j}^{(0)}(\eta, \xi), \mathrm{H}_{j}^{(1)}(\eta, \xi), \mathrm{H}_{j}^{(2)}(\eta, \xi), \ldots$, in the power series of $(62)$.

Coefficient of $\delta^{0}$

$$
\frac{\partial}{\partial \eta}\left(\eta \frac{\partial}{\partial \eta} \mathrm{H}_{j}^{(0)}(\eta, \xi)\right)+\left(\frac{\varepsilon_{j} s^{2} k_{0}^{2}}{4} \eta-\frac{1}{4 \eta}-\zeta_{u}^{(0)}(\xi)-\frac{s^{2} \beta_{j}^{2}}{4} \xi\right) \mathrm{H}_{j}^{(0)}(\eta, \xi)=0, j=1,2,(67)
$$

Coefficient of $\delta^{1}$

$$
\frac{\partial}{\partial \eta}\left(\eta \frac{\partial}{\partial \eta} \mathrm{H}_{j}^{(1)}(\eta, \xi)\right)+\left(\frac{\varepsilon_{j} s^{2} k_{0}^{2}}{4} \eta-\frac{1}{4 \eta}-\zeta_{u}^{(0)}(\xi)-\frac{s^{2} \beta_{j}^{2}}{4} \xi\right) \mathrm{H}_{j}^{(1)}(\eta, \xi)=-N_{j}^{(1)}(\eta, \xi), j=1,2,
$$

with the nonhomogeneous term

$$
N_{j}^{(1)}(\eta, \xi)=\frac{\partial^{2}}{\partial \xi^{2}} \mathrm{H}_{j}^{(0)}(\eta, \xi)+\left(1+\frac{2 \xi}{\Xi_{u}^{(0)}(\xi)} \frac{\partial}{\partial \xi} \Xi_{u}^{(0)}(\xi)\right) \frac{\partial}{\partial \xi} \mathrm{H}_{j}^{(0)}(\eta, \xi)-\zeta_{u}^{(1)}(\xi) \mathrm{H}_{j}^{(0)}(\eta, \xi), j=1,2
$$

Coefficient of $\delta^{2}$

$$
\frac{\partial}{\partial \eta}\left(\eta \frac{\partial}{\partial \eta} \mathrm{H}_{j}^{(2)}(\eta, \xi)\right)+\left(\frac{\varepsilon_{j} s^{2} k_{0}^{2}}{4} \eta-\frac{1}{4 \eta}-\zeta_{u}^{(0)}(\xi)-\frac{s^{2} \beta_{j}^{2}}{4} \xi\right) \mathrm{H}_{j}^{(2)}(\eta, \xi)=-N_{j}^{(2)}(\eta, \xi), j=1,2
$$

with the nonhomogeneous term

$$
\begin{aligned}
& N_{j}^{(2)}(\eta, \xi)=\frac{\partial^{2}}{\partial \xi^{2}} \mathrm{H}_{j}^{(1)}(\eta, \xi)+\left(1+\frac{2 \xi}{\Xi_{u}^{(0)}(\xi)} \frac{\partial}{\partial \xi} \Xi_{u}^{(0)}(\xi)\right) \frac{\partial}{\partial \xi} \mathrm{H}_{j}^{(1)}(\eta, \xi) \\
& +\frac{2 \xi}{\Xi_{u}^{(0)}(\xi)} \frac{\partial}{\partial \xi} \Xi_{u}^{(1)}(\xi) \frac{\partial}{\partial \xi} \mathrm{H}_{j}^{(0)}(\eta, \xi)-\frac{2 \xi \Xi_{u}^{(1)}(\xi)}{\left\{\Xi_{u}^{(0)}(\xi)\right\}^{2}} \frac{\partial}{\partial \xi} \Xi_{u}^{(0)}(\xi) \frac{\partial}{\partial \xi} \mathrm{H}_{j}^{(0)}(\eta, \xi) \\
& -\zeta_{u}^{(1)}(\xi) \mathrm{H}_{j}^{(1)}(\eta, \xi)-\zeta_{u}^{(2)}(\xi) \mathrm{H}_{j}^{(0)}(\eta, \xi), \quad j=1,2,
\end{aligned}
$$

etc.

By setting $\eta=\eta_{0}$ in (62) and using (9), we obtain

$$
\mathrm{H}_{j}\left(\eta_{0}, \xi\right)=\mathrm{H}_{j}^{(0)}\left(\eta_{0}, \xi\right)+\delta \mathrm{H}_{j}^{(1)}\left(\eta_{0}, \xi\right)+\delta^{2} \mathrm{H}_{j}^{(2)}\left(\eta_{0}, \xi\right)+\cdots=1, \quad j=1,2,
$$

which leads to the following Dirichlet boundary conditions

$$
\mathrm{H}_{j}^{(0)}\left(\eta_{0}, \xi\right)=1, \quad \mathrm{H}_{j}^{(1)}\left(\eta_{0}, \xi\right)=0, \quad \mathrm{H}_{j}^{(2)}\left(\eta_{0}, \xi\right)=0, \ldots \quad j=1,2
$$

by setting the coefficients of the powers of $\delta$ equal to each other in (72). In a similar manner, by substituting (62) into (58) and setting the coefficients of the powers of $\delta$ equal to each other, we obtain the following Neumann boundary conditions: 


$$
\frac{1}{\varepsilon_{1}} \lim _{\eta \rightarrow \eta_{0}-0} \frac{\partial}{\partial \eta}\left[\mathrm{H}_{1}^{(n)}(\eta, \xi) \sqrt{\eta}\right]=\frac{1}{\varepsilon_{2}} \lim _{\eta \rightarrow \eta_{0}+0} \frac{\partial}{\partial \eta}\left[\mathrm{H}_{2}^{(n)}(\eta, \xi) \sqrt{\eta}\right], \quad \mathrm{n}=0,1,2, \ldots
$$

It must be noted that imposing both the Dirichlet (73) and Neumann boundary conditions (74) provides the Cauchy boundary conditions [51], that is, we specify the values and normal derivatives of the functions $\mathrm{H}_{j}^{(0)}(\eta, \xi), \mathrm{H}_{j}^{(1)}(\eta, \xi), \mathrm{H}_{j}^{(2)}(\eta, \xi), \ldots$ in the power series (62) along the metal-dielectric boundary $\left(\eta=\eta_{0}\right)$.

Perturbation methods should be applied to the unified radial equation (39). By substituting (63) and (64) into (39) and equating the coefficients of like powers of $\delta$ on both the sides, we obtain the following system of equations for $\Xi_{u}^{(0)}(\xi), \Xi_{u}^{(1)}(\xi)$, $\Xi_{u}^{(2)}(\xi), \ldots$, in the power series (64).

Coefficient of $\delta^{0}$

$$
\frac{\partial^{2}}{\partial \xi^{2}} \Xi_{u}^{(0)}(\xi)+\frac{1}{\xi} \frac{\partial}{\partial \xi} \Xi_{u}^{(0)}(\xi)+\left(\frac{s^{2} k_{p}^{2}}{4}+\frac{\zeta_{u}^{(0)}(\xi)}{\xi}-\frac{1}{4 \xi^{2}}\right) \Xi_{u}^{(0)}(\xi)=0 \quad(0 \leq \xi<\infty),
$$

Coefficient of $\delta^{1}$

$$
\begin{aligned}
& \frac{\partial^{2}}{\partial \xi^{2}} \Xi_{u}^{(1)}(\xi)+\frac{1}{\xi} \frac{\partial}{\partial \xi} \Xi_{u}^{(1)}(\xi)+\left(\frac{s^{2} k_{p}^{2}}{4}+\frac{\zeta_{u}^{(0)}(\xi)}{\xi}-\frac{1}{4 \xi^{2}}\right) \Xi_{u}^{(1)}(\xi) \\
& =-\frac{\zeta_{u}^{(1)}(\xi)}{\xi} \Xi_{u}^{(0)}(\xi) \quad(0 \leq \xi<\infty),
\end{aligned}
$$

Coefficient of $\delta^{2}$

$$
\begin{aligned}
& \frac{\partial^{2}}{\partial \xi^{2}} \Xi_{u}^{(2)}(\xi)+\frac{1}{\xi} \frac{\partial}{\partial \xi} \Xi_{u}^{(2)}(\xi)+\left(\frac{s^{2} k_{p}^{2}}{4}+\frac{\zeta_{u}^{(0)}(\xi)}{\xi}-\frac{1}{4 \xi^{2}}\right) \Xi_{u}^{(2)}(\xi) \\
& =-\frac{\zeta_{u}^{(1)}(\xi)}{\xi} \Xi_{u}^{(1)}(\xi)-\frac{\zeta_{u}^{(2)}(\xi)}{\xi} \Xi_{u}^{(0)}(\xi) \quad(0 \leq \xi<\infty),
\end{aligned}
$$

etc.

By substituting (64) into (49), and setting the coefficients of the powers of $\delta$ equal to each other, we obtain the following Dirichlet boundary conditions:

$$
\begin{gathered}
\Xi_{u}^{(0)}(\xi) \sim \frac{2 H_{0}^{\prime}}{\pi \xi^{1 / 2}} \quad(\xi \rightarrow 0+) \\
\Xi_{u}^{(1)}(\xi)=\Xi_{u}^{(2)}(\xi)=\cdots=0 \quad(\xi \rightarrow 0+) .
\end{gathered}
$$

By substituting (64), (65) and (66) into (43), and setting the coefficients of the powers of $\delta$ equal to each other, we obtain the following relations: 


$$
\Xi_{u}^{(n)}(\xi)=\Xi_{\text {in }}^{(n)}(\xi)+\Xi_{\text {out }}^{(n)}(\xi), \quad n=0,1,2, \ldots .
$$

By substituting (65) and (66) into (44) and setting the coefficients of the powers of $\delta$ equal to each other, we obtain the following Dirichlet boundary conditions:

$$
\lim _{\xi \rightarrow 0+} \Xi_{\text {in }}^{(n)}(\xi)=\lim _{\xi \rightarrow 0+} \Xi_{\text {out }}^{(n)}(\xi), \quad n=0,1,2, \ldots
$$

Summing up the points we discussed in this section, we arrive at the following sequence of problems from which we can obtain the function sets $\left\{\zeta_{u}^{(n)}(\xi), \Xi_{u}^{(n)}(\xi), \mathrm{H}_{1}^{(n)}(\eta, \xi), \mathrm{H}_{2}^{(n)}(\eta, \xi)\right\}$ for $\mathrm{n}=0,1,2, \ldots$ in sequence:

$$
P_{0}\left\{\begin{array}{c}
\frac{\partial}{\partial \eta}\left(\eta \frac{\partial}{\partial \eta} \mathrm{H}_{j}^{(0)}(\eta, \xi)\right)+\left(\frac{\varepsilon_{\mathrm{j}} \mathrm{s}^{2} \mathrm{k}_{0}^{2}}{4} \eta-\frac{1}{4 \eta}-\zeta_{u}^{(0)}(\xi)-\frac{\mathrm{s}^{2} \beta_{\mathrm{j}}^{2}}{4} \xi\right) \mathrm{H}_{j}^{(0)}(\eta, \xi)=0, j=1,2 \\
\mathrm{H}_{j}^{(0)}\left(\eta_{0}, \xi\right)=1, \quad j=1,2 \\
\frac{1}{\varepsilon_{1}} \lim _{\eta \rightarrow \eta_{0}-0} \frac{\partial}{\partial \eta}\left[\mathrm{H}_{1}^{(0)}(\eta, \xi) \sqrt{\eta}\right]=\frac{1}{\varepsilon_{2}} \lim _{\eta \rightarrow \eta_{0}+0} \frac{\partial}{\partial \eta}\left[\mathrm{H}_{2}^{(0)}(\eta, \xi) \sqrt{\eta}\right] \\
\frac{\partial^{2}}{\partial \xi^{2}} \Xi_{u}^{(0)}(\xi)+\frac{1}{\xi} \frac{\partial}{\partial \xi} \Xi_{u}^{(0)}(\xi)+\left(\frac{s^{2} k_{p}^{2}}{4}+\frac{\zeta_{u}^{(0)}(\xi)}{\xi}-\frac{1}{4 \xi^{2}}\right) \Xi_{u}^{(0)}(\xi)=0 \\
\left.\Xi_{u}^{(0)}(\xi)\right|_{\xi \rightarrow 0+} \sim \frac{2 H_{0}^{\prime}}{\pi \xi^{1 / 2}} \\
\lim _{\xi \rightarrow 0+} \Xi_{\text {in }}^{(0)}(\xi)=\lim _{\xi \rightarrow 0+} \Xi_{\text {out }}^{(0)}(\xi), \quad \Xi_{u}^{(0)}(\xi)=\Xi_{\text {in }}^{(0)}(\xi)+\Xi_{\text {out }}^{(0)}(\xi)
\end{array}\right.
$$

$$
P_{1}\left\{\begin{array}{c}
\frac{\partial}{\partial \eta}\left(\eta \frac{\partial}{\partial \eta} \mathrm{H}_{j}^{(1)}(\eta, \xi)\right)+\left(\frac{\varepsilon_{\mathrm{j}} \mathrm{s}^{2} \mathrm{k}_{0}^{2}}{4} \eta-\frac{1}{4 \eta}-\zeta_{u}^{(0)}(\xi)-\frac{\mathrm{s}^{2} \beta_{\mathrm{j}}^{2}}{4} \xi\right) \mathrm{H}_{j}^{(1)}(\eta, \xi)=-N_{j}^{(1)}(\eta, \xi), j=1,2 \\
\mathrm{H}_{j}^{(1)}\left(\eta_{0}, \xi\right)=0, \quad j=1,2 \\
\frac{1}{\varepsilon_{1}} \lim _{\eta \rightarrow \eta_{0}-0} \frac{\partial}{\partial \eta}\left[\mathrm{H}_{1}^{(1)}(\eta, \xi) \sqrt{\eta}\right]=\frac{1}{\varepsilon_{2}} \lim _{\eta \rightarrow \eta_{0}+0} \frac{\partial}{\partial \eta}\left[\mathrm{H}_{2}^{(1)}(\eta, \xi) \sqrt{\eta}\right] \\
\frac{\partial^{2}}{\partial \xi^{2}} \Xi_{u}^{(1)}(\xi)+\frac{1}{\xi} \frac{\partial}{\partial \xi} \Xi_{u}^{(1)}(\xi)+\left(\frac{s^{2} k_{p}^{2}}{4}+\frac{\zeta_{u}^{(0)}(\xi)}{\xi}-\frac{1}{4 \xi^{2}}\right) \Xi_{u}^{(1)}(\xi)=-\frac{\zeta_{u}^{(1)}(\xi)}{\xi} \Xi_{u}^{(0)}(\xi) \\
\left.\Xi_{u}^{(1)}(\xi)\right|_{\xi \rightarrow 0+}=0 \\
\lim _{\xi \rightarrow 0+} \Xi_{\text {in }}^{(1)}(\xi)=\lim _{\xi \rightarrow 0+} \Xi_{\text {out }}^{(1)}(\xi), \quad \Xi_{u}^{(1)}(\xi)=\Xi_{\text {in }}^{(1)}(\xi)+\Xi_{\text {out }}^{(1)}(\xi)
\end{array}\right.
$$




$$
P_{2}\left\{\begin{array}{c}
\frac{\partial}{\partial \eta}\left(\eta \frac{\partial}{\partial \eta} \mathrm{H}_{j}^{(2)}(\eta, \xi)\right)+\left(\frac{\varepsilon_{\mathrm{j}} \mathrm{s}^{2} \mathrm{k}_{0}^{2}}{4} \eta-\frac{1}{4 \eta}-\zeta_{u}^{(0)}(\xi)-\frac{\mathrm{s}^{2} \beta_{\mathrm{j}}^{2}}{4} \xi\right) \mathrm{H}_{j}^{(2)}(\eta, \xi)=-N_{j}^{(2)}(\eta, \xi), j=1,2 \\
\mathrm{H}_{j}^{(2)}\left(\eta_{0}, \xi\right)=0, \quad j=1,2 \\
\frac{1}{\varepsilon_{1}} \lim _{\eta \rightarrow \eta_{0}-0} \frac{\partial}{\partial \eta}\left[\mathrm{H}_{1}^{(2)}(\eta, \xi) \sqrt{\eta}\right]=\frac{1}{\varepsilon_{2}} \lim _{\eta \rightarrow \eta_{0}+0} \frac{\partial}{\partial \eta}\left[\mathrm{H}_{2}^{(2)}(\eta, \xi) \sqrt{\eta}\right] \\
\frac{\partial^{2}}{\partial \xi^{2}} \Xi_{u}^{(2)}(\xi)+\frac{1}{\xi} \frac{\partial}{\partial \xi} \Xi_{u}^{(2)}(\xi)+\left(\frac{s^{2} k_{p}^{2}}{4}+\frac{\zeta_{u}^{(0)}(\xi)}{\xi}-\frac{1}{4 \xi^{2}}\right) \Xi_{u}^{(2)}(\xi)=-\frac{\zeta_{u}^{(1)}(\xi)}{\xi} \Xi_{u}^{(1)}(\xi)-\frac{\zeta_{u}^{(2)}(\xi)}{\xi} \Xi_{u}^{(0)}(\xi) \\
\left.\Xi_{u}^{(2)}(\xi)\right|_{\xi \rightarrow 0+}=0 \\
\lim _{\xi \rightarrow 0+} \Xi_{i n}^{(2)}(\xi)=\lim _{\xi \rightarrow 0+} \Xi_{\text {out }}^{(2)}(\xi), \quad \Xi_{u}^{(2)}(\xi)=\Xi_{\text {in }}^{(2)}(\xi)+\Xi_{\text {out }}^{(2)}(\xi)
\end{array}\right.
$$

etc. 


\section{Zeroth-order approximate solutions for the metallic hollow paraboloid}

\subsection{Extended angular functions of the zeroth order for the metallic hollow paraboloid}

The zeroth-order extended angular equations (67) can be reduced to confluent hypergeometric equations [45, 46, 52-54] whose solutions are expressed by several kinds of notations, depending upon the given parameters, such as Kummer, Whittaker, Coulomb wave, and parabolic cylinder functions. Selecting a suitable notation of confluent hypergeometric functions is important for rapidly determining the asymptotic behaviours of the functions for the given parameters.

Hereafter, we use notations of metallic permittivity $\varepsilon_{m}<0$ and dielectric permittivity $\varepsilon_{d}>0$. For the metallic hollow paraboloid, we assume $\varepsilon_{1}=\varepsilon_{d}$ and $\varepsilon_{2}=\varepsilon_{m}$. By substituting $\varepsilon_{1}=\varepsilon_{d}$ into the zeroth-order extended angular equations (67) for $j=1$, we obtain

$$
\frac{\partial}{\partial \eta}\left(\eta \frac{\partial}{\partial \eta} \mathrm{H}_{1}^{(0)}(\eta, \xi)\right)+\left(\frac{\varepsilon_{d} s^{2} k_{0}^{2}}{4} \eta-\frac{1}{4 \eta}-\zeta_{u}^{(0)}(\xi)-\frac{s^{2} \beta_{d}^{2}}{4} \xi\right) \mathrm{H}_{1}^{(0)}(\eta, \xi)=0 \quad\left(0 \leq \eta \leq \eta_{0}\right)
$$

where

$$
\beta_{d}=\sqrt{k_{p}^{2}-\varepsilon_{d} k_{0}^{2}}
$$

Equation (85) can be transformed into the following Coulomb wave equation (see Appendix B):

$$
\frac{\partial^{2}}{\partial \eta_{d}^{2}} Y\left(\eta_{d}, \xi\right)+\left(1-\frac{2 \kappa_{d}(\xi)}{\eta_{d}}\right) Y\left(\eta_{d}, \xi\right)=0
$$

with

$$
\kappa_{d}(\xi)=\frac{\zeta_{u}^{(0)}(\xi)+\frac{s^{2} \beta_{d}^{2}}{4} \xi}{s k_{0} \varepsilon_{d}^{1 / 2}}
$$

by setting

$$
\mathrm{H}_{1}^{(0)}(\eta, \xi)=\eta_{d}^{-1 / 2} Y\left(\eta_{d}, \xi\right)
$$

where

$$
\eta_{d}=\frac{\eta}{2} s k_{0} \varepsilon_{d}^{1 / 2}
$$


Two nontrivial linearly independent particular solutions to (87) are expressed by

$$
Y\left(\eta_{d}, \xi\right)=F_{0}\left(\kappa_{d}(\xi), \eta_{d}\right), \quad G_{0}\left(\kappa_{d}(\xi), \eta_{d}\right),
$$

where $F_{0}$ and $G_{0}$ are the regular and the irregular Coulomb wave functions, respectively, with the angular momentum number being zero. Therefore, from (89) and (91), it is inferred that two linearly independent solutions to (85) are expressed by

$$
\mathrm{H}_{1}^{(0)}(\eta, \xi)=\eta_{d}^{-1 / 2} F_{0}\left(\kappa_{d}(\xi), \eta_{d}\right), \quad \eta_{d}^{-1 / 2} G_{0}\left(\kappa_{d}(\xi), \eta_{d}\right) .
$$

By using equations (B.10) and (B.11), we obtain the asymptotic properties for the two solutions in (92), as follows:

$$
\begin{aligned}
\eta_{d}^{-1 / 2} F_{0}\left(\kappa_{d}(\xi), \eta_{d}\right) & \sim C_{0}\left(\kappa_{d}(\xi)\right) \eta_{d}^{1 / 2} & \left(\eta_{d} \rightarrow 0\right) \\
\eta_{d}^{-1 / 2} G_{0}\left(\kappa_{d}(\xi), \eta_{d}\right) & \sim \frac{1}{C_{0}\left(\kappa_{d}(\xi)\right)} \eta_{d}^{-1 / 2} & \left(\eta_{d} \rightarrow 0\right)
\end{aligned}
$$

where

$$
C_{0}\left(\kappa_{d}(\xi)\right)=\sqrt{\frac{2 \pi \kappa_{d}(\xi)}{\mathrm{e}^{2 \pi \kappa_{d}(\xi)}-1}} ;
$$

the properties are implied by (B.4). Because $\mathrm{H}_{1}^{(0)}(\eta, \xi)$ is finite as $\eta \rightarrow 0$ and satisfies the boundary condition $\mathrm{H}_{1}^{(0)}\left(\eta_{0}, \xi\right)=1$ in (73), by taking into account (93) and (94) for the two linearly independent solutions in (92), we obtain

$$
\mathrm{H}_{1}^{(0)}(\eta, \xi)=\sqrt{\eta_{0} / \eta} F_{0}\left(\kappa_{d}(\xi), \frac{\eta}{2} s k_{0} \varepsilon_{d}^{1 / 2}\right) / F_{0}\left(\kappa_{d}(\xi), \frac{\eta_{0}}{2} s k_{0} \varepsilon_{d}^{1 / 2}\right), 0 \leq \eta \leq \eta_{0} .
$$

In a similar manner, by substituting $\varepsilon_{2}=\varepsilon_{\mathrm{m}}$ into (67) for $j=2$, we obtain

$$
\frac{\partial}{\partial \eta}\left(\eta \frac{\partial}{\partial \eta} \mathrm{H}_{2}^{(0)}(\eta, \xi)\right)+\left(\frac{-\left|\varepsilon_{m}\right| s^{2} k_{0}^{2}}{4} \eta-\frac{1}{4 \eta}-\zeta_{u}^{(0)}(\xi)-\frac{s^{2} \beta_{m}^{2}}{4} \xi\right) \mathrm{H}_{2}^{(0)}(\eta, \xi)=0 \quad\left(\eta_{0} \leq \eta<\infty\right)
$$

where

$$
\beta_{m}=\sqrt{k_{p}^{2}-\varepsilon_{m} k_{0}^{2}}
$$

Equation (97) can be transformed into the following Whittaker's equation

$$
\frac{\partial^{2}}{\partial \eta_{m}{ }^{2}} Z\left(\eta_{m}, \xi\right)+\left[-\frac{1}{4}+\frac{\kappa_{m}(\xi)}{\eta_{m}}+\frac{\frac{1}{4}-\left(\frac{1}{2}\right)^{2}}{\eta_{m}{ }^{2}}\right] Z\left(\eta_{m}, \xi\right)=0
$$

with 


$$
\kappa_{m}(\xi)=-\frac{\zeta_{u}^{(0)}(\xi)+\frac{s^{2} \beta_{m}^{2}}{4} \xi}{s k_{0}\left|\varepsilon_{m}\right|^{1 / 2}}
$$

by setting

$$
\mathrm{H}_{2}^{(0)}(\eta, \xi)=\eta_{m}^{-1 / 2} Z\left(\eta_{m}, \xi\right)
$$

where

$$
\eta_{m}=\eta s k_{0}\left|\varepsilon_{m}\right|^{1 / 2}
$$

Two nontrivial linearly independent particular solutions to (99) are expressed by

$$
Z\left(\eta_{m}, \xi\right)=M_{\kappa_{m}(\xi), 1 / 2}\left(\eta_{m}\right), \quad W_{\kappa_{m}(\xi), 1 / 2}\left(\eta_{m}\right),
$$

where $M_{\kappa_{m}(\xi), 1 / 2}$ and $W_{\kappa_{m}(\xi), 1 / 2}$ denote the Whittaker functions of the first and second kinds, respectively, and they are defined by (B.8) and (B.7), respectively. Therefore, from (101) and (103), it is observed that two linearly independent solutions to (99) are expressed by

$$
\mathrm{H}_{2}^{(0)}(\eta, \xi)=\eta_{m}{ }^{-1 / 2} M_{\kappa_{m}(\xi), 1 / 2}\left(\eta_{m}\right), \quad \eta_{m}{ }^{-1 / 2} W_{\kappa_{m}(\xi), 1 / 2}\left(\eta_{m}\right)
$$

By using the asymptotic formulas $[53,55,56]$

$$
\begin{array}{ll}
M_{\kappa, \mu}(z) \sim e^{z / 2} z^{-\kappa} \frac{\Gamma(2 \mu+1)}{\Gamma\left(\mu-\kappa+\frac{1}{2}\right)} & \left(z \rightarrow \infty,|\arg z|<\frac{1}{2} \pi\right) \\
W_{\kappa, \mu}(z) \sim e^{-z / 2} z^{\kappa} & \left(z \rightarrow \infty,|\arg z|<\frac{3}{2} \pi\right),
\end{array}
$$

we obtain the asymptotic properties for the two solutions in (104), as follows:

$$
\begin{aligned}
\eta_{m}{ }^{-1 / 2} M_{\kappa_{m}(\xi), 1 / 2}\left(\eta_{m}\right) \sim e^{\eta_{m} / 2} \eta_{m}{ }^{-1 / 2-\kappa_{m}(\xi)} \frac{1}{\Gamma\left(1-\kappa_{m}(\xi)\right)} & & \left(\eta_{m} \rightarrow \infty\right) \\
\eta_{m}{ }^{-1 / 2} W_{\kappa_{m}(\xi), 1 / 2}\left(\eta_{m}\right) \sim e^{-\eta_{m} / 2} \eta_{m}{ }^{-1 / 2+\kappa_{m}(\xi)} & & \left(\eta_{m} \rightarrow \infty\right) .
\end{aligned}
$$

Because $\mathrm{H}_{2}^{(0)}(\eta, \xi)$ tends to approach zero as $\eta \rightarrow \infty$ and satisfies the boundary condition $\mathrm{H}_{2}^{(0)}\left(\eta_{0}, \xi\right)=1$ in (73), by taking into account (107) and (108) for the two linearly independent solutions in (104), we obtain

$$
\mathrm{H}_{2}^{(0)}(\eta, \xi)=\sqrt{\eta_{0} / \eta} W_{\kappa_{m}(\xi), 1 / 2}\left(\eta s k_{0}\left|\varepsilon_{m}\right|^{1 / 2}\right) / W_{\kappa_{m}(\xi), 1 / 2}\left(\eta_{0} s k_{0}\left|\varepsilon_{m}\right|^{1 / 2}\right), \quad \eta_{0} \leq \eta<\infty .(109)
$$


6.2 Characteristic equation for determining the unified separation quantity of the zeroth order for the metallic hollow paraboloid

By substituting (96) and (109) into (58) for $\varepsilon_{1}=\varepsilon_{d}$ and $\varepsilon_{2}=\varepsilon_{m}$, we obtain

$$
\frac{1}{2 \sqrt{\varepsilon_{d}}} \frac{F_{0}^{\prime}\left(\kappa_{d}(\xi), \frac{\eta_{0}}{2} s k_{0} \varepsilon_{d}^{1 / 2}\right)}{F_{0}\left(\kappa_{d}(\xi), \frac{\eta_{0}}{2} s k_{0} \varepsilon_{d}^{1 / 2}\right)}=-\frac{1}{\sqrt{\left|\varepsilon_{m}\right|}} \frac{W_{\kappa_{m}(\xi), 1 / 2}^{\prime}\left(\eta_{0} s k_{0}\left|\varepsilon_{m}\right|^{1 / 2}\right)}{W_{\kappa_{m}(\xi), 1 / 2}\left(\eta_{0} s k_{0}\left|\varepsilon_{m}\right|^{1 / 2}\right)}
$$

where we use notations of $F_{L}^{\prime}(\eta, \rho)=\partial F_{L}(\eta, \rho) / \partial \rho$ and $W_{\kappa, \mu}^{\prime}(z)=\partial W_{\kappa, \mu}(z) / \partial z$. Equation (110) is the characteristic equation for determining the zeroth-order unified separation quantity $\zeta_{u}^{(0)}(\xi)$ for the metallic hollow paraboloid.

Apparently, it is very difficult to solve (110) for $\zeta_{u}^{(0)}(\xi)$, analytically. In this case, we choose to solve it numerically. In numerical calculations, we use the dielectric permittivity $\varepsilon_{d}=1$ and the metallic permittivity $\varepsilon_{m}=-20$ by assuming that the dielectric matter is air with a permittivity of $\varepsilon_{d}=1$ and the metallic matter is gold with a permittivity of $\varepsilon_{m}=-20.6+1.57 \mathrm{i}$ at the $750-\mathrm{nm}$ wavelength [57]; the imaginary part of $\varepsilon_{m}$ is significantly smaller than the real part and thus can be ignored for the sake of simplicity.

In (110), the scale factor $s$ is arbitrary and remains undetermined. We set

$$
s=\lambda_{0},
$$

where $\lambda_{0}(=2 \pi \mathrm{c} / \omega)$ denotes the wavelength in vacuum. Subsequently, we obtain $s k_{0}=2 \pi$, which can be substituted into (110).

Figure 3 shows $\zeta_{u}^{(0)}(0)$ as a function of $\eta_{0}$, which is obtained by numerically solving (110) for $\zeta_{u}^{(0)}(0)$ under the conditions $\xi=0, \varepsilon_{m}=-20, \varepsilon_{d}=1$, and $s k_{0}=2 \pi$. In figure 3 , the specific value of $\eta_{0}$ for $\zeta_{u}^{(0)}(0)=0$ can be obtained analytically. By substituting $\xi=0, \zeta_{u}^{(0)}(0)=0$ and $s k_{0}=2 \pi$ into (110), we obtain

$$
\frac{1}{2 \sqrt{\varepsilon_{\mathrm{d}}}} \frac{F_{0}^{\prime}\left(0, \pi \eta_{0} \varepsilon_{d}^{1 / 2}\right)}{F_{0}\left(0, \pi \eta_{0} \varepsilon_{d}^{1 / 2}\right)}=-\frac{1}{\sqrt{\left|\varepsilon_{\mathrm{m}}\right|}} \frac{W_{0,1 / 2}^{\prime}\left(2 \pi \eta_{0}\left|\varepsilon_{m}\right|^{1 / 2}\right)}{W_{0,1 / 2}\left(2 \pi \eta_{0}\left|\varepsilon_{m}\right|^{1 / 2}\right)},
$$

which can be transformed into the form

$$
\frac{1}{\sqrt{\varepsilon_{\mathrm{d}}}} \cot \left(\pi \eta_{0} \varepsilon_{d}{ }^{1 / 2}\right)=\frac{1}{\sqrt{\left|\varepsilon_{\mathrm{m}}\right|}}
$$

by using the following formulas (see equation 14.6.4 of [53] and problem 19 of section 
9 [45])

$$
\begin{gathered}
F_{0}(0, \rho)=\sin \rho \\
W_{0,1 / 2}(z)=\sqrt{\frac{\pi}{z}} K_{1 / 2}\left(\frac{z}{2}\right)=e^{-z / 2},
\end{gathered}
$$

where $K_{1 / 2}$ represents the modified Bessel function of the second kind (also called the Macdonald function) with the order parameter being $1 / 2$. Therefore, from (113), we obtain

$$
\eta_{0}=\frac{1}{\pi \sqrt{\varepsilon_{\mathrm{d}}}} \cot ^{-1}\left(\sqrt{\frac{\varepsilon_{\mathrm{d}}}{\left|\varepsilon_{\mathrm{m}}\right|}}\right) \quad \text { for } \quad \zeta_{u}^{(0)}(0)=0
$$

From figure 3, we can easily infer that $\zeta_{u}^{(0)}(0)=0$ at $\eta_{0}=0.429976 \cdots$ from (116) calculated for $\varepsilon_{m}=-20$ and $\varepsilon_{d}=1$.

It is important to point out that the parameter $\eta_{0}$ in figure 3 can be connected with the radius of curvature $\rho_{0}$ for the circular paraboloid at the apex $(x=y=0$, $\left.z=-s \eta_{0} / 2\right)$ depicted in figure 1 . According to the formula for the curvature of space curves (for examples, see section 9.1.2-6 in [58]), we obtain

$$
\begin{aligned}
\frac{1}{\rho_{0}} & =\left.\frac{\sqrt{\left[\left(\partial_{\xi} x\right)^{2}+\left(\partial_{\xi} y\right)^{2}+\left(\partial_{\xi} z\right)^{2}\right]\left[\left(\partial_{\xi \xi} x\right)^{2}+\left(\partial_{\xi \xi} y\right)^{2}+\left(\partial_{\xi \xi} z\right)^{2}\right]-\left[\partial_{\xi} x \partial_{\xi \xi} x+\partial_{\xi} y \partial_{\xi \xi} y+\partial_{\xi} z \partial_{\xi \xi} z\right]^{2}}}{\left[\left(\partial_{\xi} x\right)^{2}+\left(\partial_{\xi} y\right)^{2}+\left(\partial_{\xi} z\right)^{2}\right]^{3 / 2}}\right|_{\xi=0} \\
& =\left.\frac{1}{s} \frac{\sqrt{\eta_{0}}}{\left(\eta_{0}+\xi\right)^{3 / 2}}\right|_{\xi=0}=\frac{1}{s \eta_{0}}
\end{aligned}
$$

by regarding (1) as the space curve of a function for $\xi$. By substituting (111) into (117), we obtain

$$
\eta_{0}=\frac{\rho_{0}}{\lambda_{0}}
$$

which implies that the parameter $\eta_{0}$ in figure 3 is equal to the radius of curvature $\rho_{0}$ divided by the wavelength in vacuum $\lambda_{0}$. The relationship in (118) is very useful for practical experiments because the right-hand side of (118) is expressed on the basis of experimental conditions. 
a figure-of-merit function

From a practical viewpoint, it is not necessarily easy to solve the characteristic equation (110) for $\zeta_{u}^{(0)}(\xi)$, numerically. In figure 4 , the solid lines indicate $\zeta_{u}^{(0)}(\xi)-\zeta_{u}^{(0)}(0)$ for specific values of $\eta_{0}\left(=\rho_{0} / \lambda_{0}\right)$ under the conditions $\varepsilon_{m}=-20$, $\varepsilon_{d}=1$, and $s k_{0}=2 \pi$; the broken lines are fitting curves for the respective solid lines. It is noted that the solid lines in figure 4 cannot be obtained for the larger values of $\xi$ by employing the usual numerical methods for computing special functions, such as those currently used in commercial computer programs such as Mathematica (Wolfram Research, Inc.). For the curve fitting, taking into account the approximate behaviour of $\zeta_{u}^{(0)}(\xi)$ for large values of $\xi$ (described in Appendix C), we select a figure-of-merit function with three parameters, $p_{1}, p_{2}$, and $p_{3}$, expressed as follows:

$$
\zeta_{u}^{(0)}(\xi)-\zeta_{u}^{(0)}(0)=p_{1}\left\{1-\exp \left[-p_{2}(1-\exp (-\xi))-p_{3} \xi\right]\right\},
$$

which implies

$$
\zeta_{u}^{\prime(0)}(0)=\left.\frac{\mathrm{d}}{\mathrm{d} \xi} \zeta_{u}^{(0)}(\xi)\right|_{\xi=0}=\lim _{\xi \rightarrow \infty} \frac{\zeta_{u}^{(0)}(\xi)-\zeta_{u}^{(0)}(0)}{\xi}=p_{1}\left(p_{2}+p_{3}\right) .
$$

In table 1 , we show several values of $p_{1}, p_{2}$, and $p_{3}$ for specific values of $\eta_{0}\left(=\rho_{0} / \lambda_{0}\right)$ obtained by fitting (119) to the solid curves shown in figure 4 (some of the curves are not shown in order to maintain clarity in the diagram). For the fitting to the solid curves for the specific values of $\eta_{0}=0.8, \eta_{0}=0.9$ and $\eta_{0}=1.0$, we assume the two parameters, $p_{2}$ and $p_{3}$, to have the same values obtained for $\eta_{0}=0.7$ in table 1 because the solid curves for $\eta_{0}=0.8,0.9,1.0$ are too short to be fitted well by (119). In table 1 , we list several values of $p_{1}\left(p_{2}+p_{3}\right)$ for specific values of $\eta_{0}\left(=\rho_{0} / \lambda_{0}\right)$, which provides the values of $\zeta_{u}^{\prime(0)}(0)$ as shown in (120).

\subsection{A unified radial function of the zeroth order for the metallic hollow paraboloid}

In the metallic hollow paraboloid, the zeroth-order unified separation quantity $\zeta_{u}^{(0)}(\xi)$ determined by (110) is quite accurately estimated by the figure-of-merit function in (119). However, we cannot solve the zeroth-order unified radial equation (75) as an already-known differential equation. In this subsection, we demonstrate that

(75) roughly approximates the Coulomb wave equation through transformations. 
By using $s=\lambda_{0}$ in (111), we express the zeroth-order unified radial equation (75) as

$$
\frac{\partial^{2}}{\partial \xi^{2}} \Xi_{u}^{(0)}(\xi)+\frac{1}{\xi} \frac{\partial}{\partial \xi} \Xi_{u}^{(0)}(\xi)+\left(\frac{\lambda_{0}^{2} k_{p}^{2}}{4}+\frac{\zeta_{u}^{(0)}(\xi)}{\xi}-\frac{1}{4 \xi^{2}}\right) \Xi_{u}^{(0)}(\xi)=0 .
$$

By defining the modified wave number of SPPs in the planar geometry, $\mathrm{k}_{m p}(\xi)$, as

$$
\mathrm{k}_{m p}(\xi)=\frac{2}{\lambda_{0}} \sqrt{\frac{\lambda_{0}^{2} \mathrm{k}_{p}^{2}}{4}+\frac{\zeta_{u}^{(0)}(\xi)-\zeta_{u}^{(0)}(0)}{\xi}}
$$

or

$$
\frac{\lambda_{0}^{2}\left\{\mathrm{k}_{m p}(\xi)\right\}^{2}}{4}=\frac{\lambda_{0}^{2} \mathrm{k}_{p}^{2}}{4}+\frac{\zeta_{u}^{(0)}(\xi)-\zeta_{u}^{(0)}(0)}{\xi}
$$

we can express (121) as

$$
\frac{\partial^{2}}{\partial \xi^{2}} \Xi_{u}^{(0)}(\xi)+\frac{1}{\xi} \frac{\partial}{\partial \xi} \Xi_{u}^{(0)}(\xi)+\left\{\frac{\lambda_{0}^{2}\left[k_{m p}(\xi)\right]^{2}}{4}+\frac{\zeta_{u}^{(0)}(0)}{\xi}-\frac{1}{4 \xi^{2}}\right\} \Xi_{u}^{(0)}(\xi)=0
$$

which can be transformed into the Coulomb wave equation if $k_{m p}(\xi)$ is constant. We solve (124) under the rough approximation

$$
k_{m p}(\xi) \approx k_{m p}(0)
$$

in which (124) approximates

$$
\frac{\partial^{2}}{\partial \xi^{2}} \Xi_{u}^{(0)}(\xi)+\frac{1}{\xi} \frac{\partial}{\partial \xi} \Xi_{u}^{(0)}(\xi)+\left\{\frac{\lambda_{0}^{2}\left[k_{m p}(0)\right]^{2}}{4}+\frac{\zeta_{u}^{(0)}(0)}{\xi}-\frac{1}{4 \xi^{2}}\right\} \Xi_{u}^{(0)}(\xi)=0
$$

where

$$
k_{m p}(0)=\frac{2}{\lambda_{0}} \sqrt{\frac{\lambda_{0}^{2} k_{p}^{2}}{4}+\zeta_{u}^{\prime(0)}(0)} .
$$

from (122). The rough approximation in (125) can be numerically estimated by the following ratio:

$$
\frac{k_{m p}(\xi)}{k_{m p}(0)}=\frac{\sqrt{\frac{\lambda_{0}^{2} k_{p}^{2}}{4}+\frac{p_{1}\left\{1-\exp \left[-p_{2}(1-\exp (-\xi))-p_{3} \xi\right]\right\}}{\xi}}}{\sqrt{\frac{\lambda_{0}^{2} k_{p}^{2}}{4}+p_{1}\left(p_{2}+p_{3}\right)}}
$$

which can be obtained from (119), (120), (122) and (127). By using the values of $p_{1}$, $p_{2}$, and $p_{3}$ in table 1 for (128), we can create graphs of the ratio $k_{m p}(\xi) / k_{m p}(0)$, as shown in figure 5 . It is shown in figure 5 that (125) is better satisfied with an increasing 
value of $\eta_{0}\left(=\rho_{0} / \lambda_{0}\right)$ and with a decreasing value of $\xi$ to zero. Numerically, (125) is reasonably satisfied in such a manner that $k_{m p}(\xi) / k_{m p}(0)$ at $\xi=14$ for $\eta_{0}=0.1$, $0.2,0.3,0.5$, and 1.0 are calculated to be $0.918,0.953,0.967,0.979$, and 0.985 , respectively, as typical values shown in figure 5 .

The approximated differential equation (126) of the zero-order unified radial equation can be transformed into the following Coulomb wave equation (see Appendix B):

$$
\frac{\partial^{2}}{\partial \bar{\xi}^{2}} X+\left(1-\frac{2 \gamma}{\bar{\xi}}\right) X=0
$$

with

$$
\gamma=-\frac{\zeta_{u}^{(0)}(0)}{\lambda_{0} k_{m p}(0)}
$$

by setting

$$
\Xi_{u}^{(0)}(\xi)=\bar{\xi}^{-1 / 2} X
$$

where

$$
\bar{\xi}=\frac{\lambda_{0} k_{m p}(0)}{2} \xi
$$

For (129), outgoing and incoming Coulomb wave functions (see equations (B.17) and (B.18)) are described by

$$
H_{0}^{+}(\gamma, \bar{\xi})=G_{0}(\gamma, \bar{\xi})+\mathrm{i} F_{0}(\gamma, \bar{\xi})
$$

and

$$
H_{0}^{-}(\gamma, \bar{\xi})=G_{0}(\gamma, \bar{\xi})-\mathrm{i} F_{0}(\gamma, \bar{\xi})
$$

respectively, where $F_{0}$ and $G_{0}$ denote the regular and the irregular Coulomb wave functions, respectively, with the angular momentum number being zero. Therefore, taking into account (131) together with (133) and (134), outgoing and incoming solutions for (126) are expressed by $\bar{\xi}^{-1 / 2} H_{0}^{+}(\gamma, \bar{\xi})$ and $\bar{\xi}^{-1 / 2} H_{0}^{-}(\gamma, \bar{\xi})$, respectively. For the limit $\xi \rightarrow \infty$, from the asymptotic formulas (B.19) and (B.20), it is inferred that $\bar{\xi}^{-1 / 2} H_{0}^{+}(\gamma, \bar{\xi}) \sim\left(\frac{\lambda_{0} k_{m p}(0)}{2}\right)^{-1 / 2} \xi^{-1 / 2} \exp \left\{i\left[\frac{\lambda_{0} k_{m p}(0)}{2} \xi-\ln \left(\lambda_{0} k_{m p}(0) \xi\right)+\sigma_{0}(\gamma)\right]\right\} \quad(\xi \rightarrow \infty)$ 


$$
\bar{\xi}^{-1 / 2} H_{0}^{-}(\gamma, \bar{\xi}) \sim\left(\frac{\lambda_{0} k_{m p}(0)}{2}\right)^{-1 / 2} \xi^{-1 / 2} \exp \left\{-i\left[\frac{\lambda_{0} k_{m p}(0)}{2} \xi-\ln \left(\lambda_{0} k_{m p}(0) \xi\right)+\sigma_{0}(\gamma)\right]\right\}(\xi \rightarrow \infty)
$$

For the limit $\xi \rightarrow 0+$, from the asymptotic formulas (B.21) and (B.22), it is inferred that

$$
\begin{array}{ll}
\bar{\xi}^{-1 / 2} H_{0}^{+}(\gamma, \bar{\xi}) \sim\left(\frac{\lambda_{0} k_{m p}(0)}{2}\right)^{-1 / 2} \xi^{-1 / 2} \frac{1}{C_{0}(\gamma)} & (\xi \rightarrow 0+) \\
\bar{\xi}^{-1 / 2} H_{0}^{-}(\gamma, \bar{\xi}) \sim\left(\frac{\lambda_{0} k_{m p}(0)}{2}\right)^{-1 / 2} \xi^{-1 / 2} \frac{1}{C_{0}(\gamma)} & (\xi \rightarrow 0+)
\end{array}
$$

where (132) is employed, and equation (B.4) implies

$$
C_{0}(\gamma)=\sqrt{\frac{2 \pi \gamma}{\mathrm{e}^{2 \pi \gamma}-1}} .
$$

Considering (81) for (137) and (138), we select

$$
\begin{aligned}
& \Xi_{\text {out }}^{(0)}(\xi)=H_{0}^{\prime \prime} \xi^{-1 / 2} H_{0}^{+}\left(\gamma, \frac{\lambda_{0} k_{m p}(0)}{2} \xi\right) \\
& \Xi_{\text {in }}^{(0)}(\xi)=H_{0}^{\prime \prime} \xi^{-1 / 2} H_{0}^{-}\left(\gamma, \frac{\lambda_{0} k_{m p}(0)}{2} \xi\right)
\end{aligned}
$$

where

$$
H_{0}^{\prime \prime}=\frac{C_{0}(\gamma)}{\pi} H_{0}^{\prime}
$$

is obtained by taking into account (78) and (80). Finally, by using (80) for (140) and (141), we obtain

$$
\Xi_{u}^{(0)}(\xi)=2 H_{0}^{\prime \prime} \xi^{-1 / 2} G_{0}\left(\gamma, \frac{\lambda_{0} k_{m p}(0)}{2} \xi\right),
$$

which indicates neither outgoing nor incoming waves but a standing wave with the free end.

It is important to point out that the parameter $\gamma$ of the irregular Coulomb wave function $G_{0}$ in (143) can be studied from the viewpoint of the Sommerfeld parameter in Coulomb wave functions, which describes the strength of interaction between two charged particles for positive energy of relative motion [62]: positive and negative 
values of the Sommerfeld parameter correspond to repulsive and attractive potentials, respectively, and the Sommerfeld parameter with a value of zero corresponds exactly to zero potential. From (130), it is inferred that $\gamma$ is positive when $\zeta_{u}^{(0)}(0)<0$, and it is negative when $\zeta_{u}^{(0)}(0)>0$. The behaviour of $\zeta_{u}^{(0)}(0)$ as a function of $\eta_{0}\left(=\rho_{0} / \lambda_{0}\right)$ for the specific condition $\varepsilon_{m}=-20$ and $\varepsilon_{d}=1$ is shown in figure 3 in which $\zeta_{u}^{(0)}(0)=0$ is satisfied for $\eta_{0}=0.429976$ calculated from (116). For values of $\eta_{0}\left(=\rho_{0} / \lambda_{0}\right)$ smaller than $\eta_{0}=0.429976$ in figure 3 , the Sommerfeld parameter $\gamma$ of Coulomb wave functions corresponds to repulsive potentials; for larger values, the Sommerfeld parameter $\gamma$ corresponds to attractive potentials. This can be understood easily by employing a geometrical approach by using the relation $\eta_{0}=\rho_{0} / \lambda_{0}$ in (118): the Sommerfeld parameter $\gamma$ of the irregular Coulomb wave function $G_{0}$ in (143) corresponds to the repulsive and attractive potentials for smaller and larger radii of curvature, respectively, at the apex of the circular hollow paraboloid. 


\section{Non-existence of zeroth-order solutions for the metallic solid paraboloid}

In this section, we prove that no plasmonic modes exist for the metallic solid paraboloid by examining (67).

For the metallic solid paraboloid, we can express $\varepsilon_{1}=\varepsilon_{m}<0$ and $\varepsilon_{2}=\varepsilon_{d}>0$. By substituting $\varepsilon_{2}=\varepsilon_{d}$ into (67) for $j=2$, we obtain

$$
\frac{\partial}{\partial \eta}\left(\eta \frac{\partial}{\partial \eta} \mathrm{H}_{2}^{(0)}(\eta, \xi)\right)+\left(\frac{\varepsilon_{d} s^{2} k_{0}^{2}}{4} \eta-\frac{1}{4 \eta}-\zeta_{u}^{(0)}(\xi)-\frac{s^{2} \beta_{d}^{2}}{4} \xi\right) \mathrm{H}_{2}^{(0)}(\eta, \xi)=0 \quad\left(\eta_{0} \leq \eta<\infty\right),
$$

which can be solved in the same manner as that employed in (85). Referring to (92), which provides two linearly independent solutions to (85), we observe that two linearly independent solutions to (144) are expressed by

$$
\mathrm{H}_{2}^{(0)}(\eta, \xi)=\eta_{d}^{-1 / 2} F_{0}\left(\kappa_{d}(\xi), \eta_{d}\right), \quad \eta_{d}^{-1 / 2} G_{0}\left(\kappa_{d}(\xi), \eta_{d}\right) .
$$

By using (B.12) and (B.13), we find that the asymptotic behaviours of the two solutions in (145) are oscillatory, with the amplitude varying as $\eta^{-1 / 2}$ for the limit of $\eta \rightarrow \infty$; this behaviour is similar to those exhibited by the outgoing and incoming solutions of the unified radial equation for $\xi \rightarrow \infty$ in (135) and (136). This clearly indicates that the two linearly independent solutions in (145) are unsuitable for composition of the zeroth-order extended angular function for $\eta_{0} \leq \eta<\infty$ because their behaviour does not to localization but to propagation. Therefore, we can arrive at a very interesting conclusion that plasmonic modes do not exist for the metallic solid paraboloid. 


\section{First-order perturbation solutions for the metallic hollow paraboloid}

In this section, we consider the first-order perturbation solution for the metallic hollow paraboloid, by solving problem $P_{1}$ of (83). Problem $P_{1}$ is involved in the solving of nonhomogeneous ordinary differential equations (ODEs) in which homogeneous ODEs without nonhomogeneous terms are already solved in problem $P_{0}$ (82). Specific solutions to the nonhomogeneous ODEs for the unified radial and the extended angular functions can be obtained by using the variation of parameters and Green's function methods, respectively.

\subsection{Extended angular functions of the first order for the metallic hollow paraboloid}

For $P_{1}(83)$, we consider the first-order extended angular equation

$$
\frac{\partial}{\partial \eta}\left(\eta \frac{\partial}{\partial \eta} \mathrm{H}_{j}^{(1)}(\eta, \xi)\right)+\left(\frac{\varepsilon_{\mathrm{j}} s^{2} k_{0}^{2}}{4} \eta-\frac{1}{4 \eta}-\zeta_{u}^{(0)}(\xi)-\frac{s^{2} \beta_{\mathrm{j}}^{2}}{4} \xi\right) \mathrm{H}_{j}^{(1)}(\eta, \xi)=-N_{j}^{(1)}(\eta, \xi), j=1,2
$$

under the boundary condition

$$
\mathrm{H}_{j}^{(1)}\left(\eta_{0}, \xi\right)=0, \quad j=1,2 .
$$

Generally, for the self-adjoint homogeneous differential equation

$$
\frac{d}{d x}\left(p(x) \frac{d}{d x}\right) y(x)+q(x) y(x)=0, \quad a \leq x \leq b,
$$

from chapter 10.5 in [59], it is observed that the nonhomogeneous differential equation

$$
\frac{d}{d x}\left(p(x) \frac{d}{d x}\right) y(x)+q(x) y(x)=-f(x)
$$

has the solution

$$
y(x)=\int_{a}^{b} G(x, t) f(t) d t
$$

with the Green's function

$$
G(x, t)=\left\{\begin{aligned}
-\frac{u(x) v(t)}{p(t) W(t)}, & a \leq x<t \\
-\frac{u(t) v(x)}{p(t) W(t)}, & t<x \leq b
\end{aligned}\right.
$$


where $u(x)$ and $v(x)$ represent two independent solutions of the homogenous differential equation (148) that satisfy the boundary conditions at $x=a$ and $x=b$, respectively, and

$$
W(t)=u(t) v^{\prime}(t)-v(t) u^{\prime}(t)
$$

is the Wronskian.

For the dielectric region $j=1$, according to the Green's function method, the nonhomogeneous ODE (146) under the boundary condition (147) has the solution

$$
\mathrm{H}_{1}^{(1)}(\eta, \xi)=\int_{0}^{\eta_{0}} G_{1}\left(\eta, \eta^{\prime}\right) N_{1}^{(1)}(\eta, \xi) \mathrm{d} \eta^{\prime} \quad\left(0 \leq \eta \leq \eta_{0}\right)
$$

with the Green's function

$$
G\left(\eta, \eta^{\prime}\right)= \begin{cases}-\frac{u_{1}(\eta) v_{1}\left(\eta^{\prime}\right)}{p_{1}\left(\eta^{\prime}\right) W_{1}\left(\eta^{\prime}\right)}, & 0 \leq \eta<\eta^{\prime} \\ -\frac{u_{1}\left(\eta^{\prime}\right) v_{1}(\eta)}{p_{1}\left(\eta^{\prime}\right) W_{1}\left(\eta^{\prime}\right)}, & \eta^{\prime}<\eta \leq \eta_{0},\end{cases}
$$

where, from (92), we can select

$$
\begin{gathered}
u_{1}(\eta)=\sqrt{\frac{\eta_{0}}{\eta}} \frac{F_{0}\left(\kappa_{d}(\xi), \frac{\eta}{2} s k_{0} \varepsilon_{d}{ }^{1 / 2}\right)}{F_{0}\left(\kappa_{d}(\xi), \frac{\eta_{0}}{2} s k_{0} \varepsilon_{d}{ }^{1 / 2}\right)} \\
v_{1}(\eta)=\sqrt{\frac{\eta_{0}}{\eta}} \frac{F_{0}\left(\kappa_{d}(\xi), \frac{\eta}{2} s k_{0} \varepsilon_{d}{ }^{1 / 2}\right)}{F_{0}\left(\kappa_{d}(\xi), \frac{\eta_{0}}{2} s k_{0} \varepsilon_{d}{ }^{1 / 2}\right)}-\sqrt{\frac{\eta_{0}}{\eta}} \frac{G_{0}\left(\kappa_{d}(\xi), \frac{\eta}{2} s k_{0} \varepsilon_{d}{ }^{1 / 2}\right)}{G_{0}\left(\kappa_{d}(\xi), \frac{\eta_{0}}{2} s k_{0} \varepsilon_{d}{ }^{1 / 2}\right)}
\end{gathered}
$$

by taking account of $u_{1}(0)=$ finite and $v_{1}\left(\eta_{0}\right)=0$. By using equation (B.14), the Wronskian of the pair, $u_{1}(\eta)$ and $v_{1}(\eta)$, is obtained as

$$
W_{1}(\eta)=\frac{\eta_{0}}{\eta} \frac{s k_{0} \varepsilon_{d}^{1 / 2}}{2 F_{0}\left(\kappa_{d}(\xi), \frac{\eta_{0}}{2} s k_{0} \varepsilon_{d}^{1 / 2}\right) G_{0}\left(\kappa_{d}(\xi), \frac{\eta_{0}}{2} s k_{0} \varepsilon_{d}^{1 / 2}\right)},
$$

and by comparing (146) and (149), we can assume

$$
p_{1}(\eta)=\eta \text {. }
$$

It follows that 


$$
\begin{aligned}
& \mathrm{H}_{1}^{(1)}(\eta, \xi)=-\frac{\mathrm{F}_{0}\left(\kappa_{\mathrm{d}}(\xi), \frac{\eta_{0}}{2} \mathrm{sk}_{0} \varepsilon_{\mathrm{d}}{ }^{1 / 2}\right) \mathrm{G}_{0}\left(\kappa_{\mathrm{d}}(\xi), \frac{\eta_{0}}{2} s k_{0} \varepsilon_{\mathrm{d}}{ }^{1 / 2}\right)}{\frac{\eta_{0}}{2} \mathrm{sk}_{0} \varepsilon_{\mathrm{d}}{ }^{1 / 2}} \\
& \times\left[v_{1}(\eta) \int_{0}^{\eta} u_{1}\left(\eta^{\prime}\right) N_{1}^{(1)}\left(\eta^{\prime}, \xi\right) \mathrm{d} \eta^{\prime}+u_{1}(\eta) \int_{\eta}^{\eta_{0}} v_{1}\left(\eta^{\prime}\right) N_{1}^{(1)}\left(\eta^{\prime}, \xi\right) \mathrm{d} \eta^{\prime}\right] .
\end{aligned}
$$

For the dielectric region $j=2$, according to the Green's function method, the nonhomogeneous ODE (146) under the boundary condition (147) has the solution

$$
\mathrm{H}_{2}^{(1)}(\eta, \xi)=\int_{\eta_{0}}^{\infty} G_{2}\left(\eta, \eta^{\prime}\right) N_{2}^{(1)}(\eta, \xi) \mathrm{d} \eta^{\prime} \quad\left(\eta_{0} \leq \eta<\infty\right)
$$

with the Green's function

$$
G\left(\eta, \eta^{\prime}\right)= \begin{cases}-\frac{u_{2}(\eta) v_{2}\left(\eta^{\prime}\right)}{p_{2}\left(\eta^{\prime}\right) W_{2}\left(\eta^{\prime}\right)}, & \eta_{0} \leq \eta<\eta^{\prime} \\ -\frac{u_{2}\left(\eta^{\prime}\right) v_{2}(\eta)}{p_{2}\left(\eta^{\prime}\right) W_{2}\left(\eta^{\prime}\right)}, & \eta^{\prime}<\eta<\infty,\end{cases}
$$

where, from (104), we can select

$$
\begin{gathered}
u_{2}(\eta)=\sqrt{\frac{\eta_{0}}{\eta}} \frac{W_{\kappa_{m}(\xi), 1 / 2}\left(\eta s k_{0}\left|\varepsilon_{m}\right|^{1 / 2}\right)}{W_{\kappa_{m}(\xi), 1 / 2}\left(\eta_{0} s k_{0}\left|\varepsilon_{m}\right|^{1 / 2}\right)}-\sqrt{\frac{\eta_{0}}{\eta}} \frac{M_{\kappa_{m}(\xi), 1 / 2}\left(\eta s k_{0}\left|\varepsilon_{m}\right|^{1 / 2}\right)}{M_{\kappa_{m}(\xi), 1 / 2}\left(\eta_{0} s k_{0}\left|\varepsilon_{m}\right|^{1 / 2}\right)} \\
v_{2}(\eta)=\sqrt{\frac{\eta_{0}}{\eta}} \frac{W_{\kappa_{m}(\xi), 1 / 2}\left(\eta s k_{0}\left|\varepsilon_{m}\right|^{1 / 2}\right)}{W_{\kappa_{m}(\xi), 1 / 2}\left(\eta_{0} s k_{0}\left|\varepsilon_{m}\right|^{1 / 2}\right)}
\end{gathered}
$$

by taking account of $u_{2}\left(\eta_{0}\right)=0$ and $v_{2}(\infty)=$ finite; by using (B.9), the Wronskian of the pair, $u_{2}(\eta)$ and $v_{2}(\eta)$, is obtained as

$$
W_{2}(\eta)=\frac{\eta_{0}}{\eta} \frac{s k_{0}\left|\varepsilon_{m}\right|^{1 / 2}}{\Gamma\left(1-\kappa_{m}(\xi)\right) M_{\kappa_{m}(\xi), 1 / 2}\left(\eta_{0} s k_{0}\left|\varepsilon_{m}\right|^{1 / 2}\right) W_{\kappa_{m}(\xi), 1 / 2}\left(\eta_{0} s k_{0}\left|\varepsilon_{m}\right|^{1 / 2}\right)},
$$

and by comparing (146) and (149), we assume

$$
p_{2}(\eta)=\eta \text {. }
$$

It follows that 


$$
\begin{aligned}
& \mathrm{H}_{2}^{(1)}(\eta, \xi)=-\frac{\Gamma\left(1-\kappa_{m}(\xi)\right) M_{\kappa_{m}(\xi), 1 / 2}\left(\eta_{0} s k_{0}\left|\varepsilon_{m}\right|^{1 / 2}\right) W_{\kappa_{m}(\xi), 1 / 2}\left(\eta_{0} s k_{0}\left|\varepsilon_{m}\right|^{1 / 2}\right)}{s k_{0}\left|\varepsilon_{m}\right|^{1 / 2}} \\
& \times\left[v_{2}(\eta) \int_{\eta_{0}}^{\eta} u_{2}\left(\eta^{\prime}\right) N_{2}^{(1)}\left(\eta^{\prime}, \xi\right) \mathrm{d} \eta^{\prime}+u_{2}(\eta) \int_{\eta}^{\infty} v_{2}\left(\eta^{\prime}\right) N_{2}^{(1)}\left(\eta^{\prime}, \xi\right) \mathrm{d} \eta^{\prime}\right]
\end{aligned}
$$

For numerical calculations, the scale factor $s$ appearing in all the equations in this subsection is replaced with the wavelength in vacuum $\lambda_{0}$, as shown in (111).

8.2 Determination of the unified separation quantity of the first order for the metallic hollow paraboloid

For problem $P_{1}$ (83), we consider the following boundary condition

$$
\frac{1}{\varepsilon_{d}} \lim _{\eta \rightarrow \eta_{0}-0} \frac{\partial}{\partial \eta}\left[\mathrm{H}_{1}^{(1)}(\eta, \xi) \sqrt{\eta}\right]=\frac{1}{\varepsilon_{m}} \lim _{\eta \rightarrow \eta_{0}+0} \frac{\partial}{\partial \eta}\left[\mathrm{H}_{2}^{(1)}(\eta, \xi) \sqrt{\eta}\right],
$$

which can be used to determine the first-order unified separation quantity $\zeta_{u}^{(1)}(\xi)$. By substituting (159) into the left-hand side of (167) and by using (B.14), we obtain

$$
\frac{1}{\varepsilon_{d}} \lim _{\eta \rightarrow \eta_{0}-0} \frac{\partial}{\partial \eta}\left[\mathrm{H}_{1}^{(1)}(\eta, \xi) \sqrt{\eta}\right]=-\frac{1}{\varepsilon_{d}} \int_{0}^{\eta_{0}} u_{1}\left(\eta^{\prime}\right) N_{1}^{(1)}\left(\eta^{\prime}, \xi\right) \mathrm{d} \eta^{\prime}
$$

By substituting (166) into the right-hand side of (167) and by using (B.9), we obtain

$$
\frac{1}{\varepsilon_{m}} \lim _{\eta \rightarrow \eta_{0}+0} \frac{\partial}{\partial \eta}\left[\mathrm{H}_{2}^{(1)}(\eta, \xi) \sqrt{\eta}\right]=\frac{1}{\varepsilon_{m}} \int_{\eta_{0}}^{\infty} v_{2}\left(\eta^{\prime}\right) N_{2}^{(1)}\left(\eta^{\prime}, \xi\right) \mathrm{d} \eta^{\prime} .
$$

By setting (168) equal to (169) according to (167), and by taking into consideration from (96), (109), (155), and (163) that $u_{1}(\eta)=\mathrm{H}_{1}^{(0)}(\eta, \xi)$ and $v_{2}(\eta)=\mathrm{H}_{2}^{(0)}(\eta, \xi)$, we obtain

$$
\frac{1}{\varepsilon_{d}} \int_{0}^{\eta_{0}} \mathrm{H}_{1}^{(0)}(\eta, \xi) N_{1}^{(1)}(\eta, \xi) \mathrm{d} \eta+\frac{1}{\varepsilon_{m}} \int_{\eta_{0}}^{\infty} \mathrm{H}_{2}^{(0)}(\eta, \xi) N_{2}^{(1)}(\eta, \xi) \mathrm{d} \eta=0 .
$$

By substituting (69) into (170) and by rearranging terms, we determine for the first-order unified separation quantity that 


$$
\zeta_{u}^{(1)}(\xi)=\frac{\left[\begin{array}{c}
\frac{1}{\varepsilon_{d}} \int_{0}^{\eta_{0}} \mathrm{H}_{1}^{(0)}(\eta, \xi) \frac{\partial^{2}}{\partial \xi^{2}} \mathrm{H}_{1}^{(0)}(\eta, \xi) \mathrm{d} \eta+\frac{1}{\varepsilon_{m}} \int_{\eta_{0}}^{\infty} \mathrm{H}_{2}^{(0)}(\eta, \xi) \frac{\partial^{2}}{\partial \xi^{2}} \mathrm{H}_{2}^{(0)}(\eta, \xi) \mathrm{d} \eta \\
+\left(1+\frac{2 \xi}{\Xi_{\mathrm{u}}^{(0)}(\xi)} \frac{\partial}{\partial \xi} \Xi_{\mathrm{u}}^{(0)}(\xi)\right)\left(\begin{array}{l}
\frac{1}{\varepsilon_{d}} \int_{0}^{\eta_{0}} \mathrm{H}_{1}^{(0)}(\eta, \xi) \frac{\partial}{\partial \xi} \mathrm{H}_{1}^{(0)}(\eta, \xi) \mathrm{d} \eta \\
+\frac{1}{\varepsilon_{m}} \int_{\eta_{0}}^{\infty} \mathrm{H}_{2}^{(0)}(\eta, \xi) \frac{\partial}{\partial \xi} \mathrm{H}_{2}^{(0)}(\eta, \xi) \mathrm{d} \eta
\end{array}\right)
\end{array}\right]}{\frac{1}{\varepsilon_{d}} \int_{0}^{\eta_{0}}\left\{\mathrm{H}_{1}^{(0)}(\eta, \xi)\right\}^{2} \mathrm{~d} \eta+\frac{1}{\varepsilon_{m}} \int_{\eta_{0}}^{\infty}\left\{\mathrm{H}_{2}^{(0)}(\eta, \xi)\right\}^{2} \mathrm{~d} \eta}
$$

\subsection{Unified radial function of the first order for the metallic hollow paraboloid}

For problem $P_{1}(83)$, here we consider the first-order unified radial equation

$$
\frac{\partial^{2}}{\partial \xi^{2}} \Xi_{u}^{(1)}(\xi)+\frac{1}{\xi} \frac{\partial}{\partial \xi} \Xi_{u}^{(1)}(\xi)+\left(\frac{s^{2} k_{p}^{2}}{4}+\frac{\zeta_{u}^{(0)}(\xi)}{\xi}-\frac{1}{4 \xi^{2}}\right) \Xi_{u}^{(1)}(\xi)=-\frac{\zeta_{u}^{(1)}(\xi)}{\xi} \Xi_{u}^{(0)}(\xi)
$$

under the boundary conditions

$$
\begin{gathered}
\left.\Xi_{u}^{(1)}(\xi)\right|_{\xi \rightarrow 0+}=0 \\
\lim _{\xi \rightarrow 0+} \Xi_{\text {in }}^{(1)}(\xi)=\lim _{\xi \rightarrow 0+} \Xi_{\text {out }}^{(1)}(\xi), \quad \Xi_{u}^{(1)}(\xi)=\Xi_{\text {in }}^{(1)}(\xi)+\Xi_{\text {out }}^{(1)}(\xi) .
\end{gathered}
$$

By using $s=\lambda_{0}$ in (111), we can rewrite (172) as

$$
\frac{\partial^{2}}{\partial \xi^{2}} \Xi_{u}^{(1)}(\xi)+\frac{1}{\xi} \frac{\partial}{\partial \xi} \Xi_{u}^{(1)}(\xi)+\left(\frac{\lambda_{0}^{2} k_{p}^{2}}{4}+\frac{\zeta_{u}^{(0)}(\xi)}{\xi}-\frac{1}{4 \xi^{2}}\right) \Xi_{u}^{(1)}(\xi)=-\frac{\zeta_{u}^{(1)}(\xi)}{\xi} \Xi_{u}^{(0)}(\xi),
$$

whose associated homogeneous ODE is the same as that in (121), which has already been solved in subsection 6.4 .

According to exercise 9.6.25 in [59], the nonhomogeneous, linear, second-order ODE

$$
\frac{d^{2} y}{d x^{2}}+P(x) \frac{d y}{d x}+Q(x) y=F(x)
$$

has the solution

$$
y(x)=c_{1} y_{1}(x)+c_{2} y_{2}(x)+y_{p}(x)
$$

where $y_{1}(x)$ and $y_{2}(x)$ represent two nontrivial linearly independent solutions to the associated homogeneous ODE 


$$
\frac{d^{2} y}{d x^{2}}+P(x) \frac{d y}{d x}+Q(x) y=0
$$

and $y_{p}(x)$ represents a particular solution expressed by

$$
y_{p}(x)=y_{2}(x) \int^{x} \frac{y_{1}(s) F(s) d s}{W\left\{y_{1}(s), y_{2}(s)\right\}}-y_{1}(x) \int^{x} \frac{y_{2}(s) F(s) d s}{W\left\{y_{1}(s), y_{2}(s)\right\}}
$$

where $W\left\{y_{1}(s), y_{2}(s)\right\}$ denotes the Wronskian of $y_{1}(s)$ and $y_{2}(s)$. The constants $c_{1}$ and $c_{2}$ in (177) and the lower limits of the two integrals in (179) are fixed according to boundary conditions. For the nonhomogeneous ODE (172), similar to how equation (121) is solved in subsection 6.4, by selecting $\xi^{-1 / 2} H_{0}^{+}\left(\gamma, \lambda_{0} k_{m p}(0) \xi / 2\right)$ and $\xi^{-1 / 2} H_{0}^{-}\left(\gamma, \lambda_{0} k_{m p}(0) \xi / 2\right)$ as two nontrivial linearly independent solutions, we obtain the Wronskian

$$
W\left\{\xi^{-1 / 2} H_{0}^{+}\left(\gamma, \frac{\lambda_{0} k_{m p}(0)}{2} \xi\right), \xi^{-1 / 2} H_{0}^{-}\left(\gamma, \frac{\lambda_{0} k_{m p}(0)}{2} \xi\right)\right\}=-i \frac{\lambda_{0} k_{m p}(0)}{\xi}
$$

by referring to (B.14). By taking into account the boundary conditions (173) and (174), we find that the solution is $\Xi_{u}^{(1)}(\xi)=\Xi_{\text {in }}^{(1)}(\xi)+\Xi_{\text {out }}^{(1)}(\xi)$, where

$$
\begin{aligned}
& \Xi_{\text {out }}^{(1)}(\xi)=\frac{\mathrm{i}}{\lambda_{0} k_{m p}(0)} \xi^{-1 / 2} H_{0}^{+}\left(\gamma, \frac{\lambda_{0} k_{m p}(0)}{2} \xi\right) \int_{0}^{\xi} \zeta_{u}^{(1)}\left(\xi^{\prime}\right) \Xi_{u}^{(0)}\left(\xi^{\prime}\right) \xi^{\prime-1 / 2} H_{0}^{-}\left(\gamma, \frac{\lambda_{0} k_{m p}(0)}{2} \xi^{\prime}\right) d \xi^{\prime} \\
& \Xi_{\text {in }}^{(1)}(\xi)=-\frac{\mathrm{i}}{\lambda_{0} k_{m p}(0)} \xi^{-1 / 2} H_{0}^{-}\left(\gamma, \frac{\lambda_{0} k_{m p}(0)}{2} \xi\right) \int_{0}^{\xi} \zeta_{u}^{(1)}\left(\xi^{\prime}\right) \Xi_{u}^{(0)}\left(\xi^{\prime}\right) \xi^{\prime-1 / 2} H_{0}^{+}\left(\gamma, \frac{\lambda_{0} k_{m p}(0)}{2} \xi^{\prime}\right) d \xi^{\prime} .
\end{aligned}
$$

The functions $\Xi_{\text {out }}^{(1)}(\xi)$ and $\Xi_{\text {in }}^{(1)}(\xi)$ correspond to the outgoing and incoming waves for $\xi \rightarrow \infty$, respectively. It must be noted that the solution can be simplified into the form

$$
\begin{aligned}
& \Xi_{u}^{(1)}(\xi)=\frac{2}{\lambda_{0} k_{m p}(0)} \xi^{-1 / 2} F_{0}\left(\gamma, \frac{\lambda_{0} k_{m p}(0)}{2} \xi\right) \int_{0}^{\xi} \zeta_{u}^{(1)}\left(\xi^{\prime}\right) \Xi_{u}^{(0)}\left(\xi^{\prime}\right) \xi^{\prime-1 / 2} G_{0}\left(\gamma, \frac{\lambda_{0} k_{m p}(0)}{2} \xi^{\prime}\right) d \xi^{\prime} \\
& -\frac{2}{\lambda_{0} k_{m p}(0)} \xi^{-1 / 2} G_{0}\left(\gamma, \frac{\lambda_{0} k_{m p}(0)}{2} \xi\right) \int_{0}^{\xi} \zeta_{u}^{(1)}\left(\xi^{\prime}\right) \Xi_{u}^{(0)}\left(\xi^{\prime}\right) \xi^{\prime-1 / 2} F_{0}\left(\gamma, \frac{\lambda_{0} k_{m p}(0)}{2} \xi^{\prime}\right) d \xi^{\prime}
\end{aligned}
$$

which indicates neither outgoing nor incoming waves but a standing wave with a free end. 
The second-order perturbation solutions can be obtained in much the same manner as the first-order perturbation solutions. We do not provide the second-order solutions in this paper in order to reduce verbosity. It is potentially useful to indicate that the second-order perturbation solutions have been provided for wedge-shaped geometry (refer to appendices C and D in [19]). 


\section{Electric field-line representation}

It is very important to understand plasmonic modes not only mathematically but also visually. In this case where the electromagnetic field has only a $\phi$-component of the magnetic field described as

$$
\mathrm{H}_{\phi}(\xi, \eta, t)=\operatorname{Re}\left[\mathrm{H}_{\phi}(\xi, \eta) \mathrm{e}^{-\mathrm{i} \omega \mathrm{t}}\right]
$$

we can easily use a field-line pattern representation of the electric field, which is graphically simpler and more intuitively sophisticated than the field-vector pattern representation.

We describe the field-line pattern representation of an electric field in detail. The tangent at an arbitrary point of electric field line indicates the direction of electric field vector $\boldsymbol{E}(\xi, \eta, t)$ at this point. This fact can be described mathematically by using a line vector element $d \boldsymbol{s}$ as follows:

$$
\boldsymbol{E}(\xi, \eta, t) \times d \boldsymbol{s}=0
$$

which can be simplified into the form

$$
\frac{d \xi}{E_{\xi}(\xi, \eta, t) \sqrt{\xi}}-\frac{d \eta}{E_{\eta}(\xi, \eta, t) \sqrt{\eta}}=0
$$

when

$$
\begin{gathered}
\boldsymbol{E}(\xi, \eta, t)=E_{\xi}(\xi, \eta, t) \boldsymbol{e}_{\xi}+E_{\eta}(\xi, \eta, t) \boldsymbol{e}_{\eta} \\
d \boldsymbol{s}=\mathrm{h}_{\xi} d \xi \boldsymbol{e}_{\xi}+\mathrm{h}_{\eta} d \eta \boldsymbol{e}_{\eta}
\end{gathered}
$$

where $\boldsymbol{e}_{\xi}$ and $\boldsymbol{e}_{\eta}$ denote the unit vectors to the $\xi$ - and $\eta$-axes, respectively; $\mathrm{h}_{\xi}$ and $\mathrm{h}_{\eta}$ denote the metric coefficients given in (A.5). For the magnetic field in (184), by using (50)-(56) with (111), we obtain the $\xi$ and $\eta$ components of the electric field as follows:

$$
\begin{aligned}
& E_{\xi}(\xi, \eta, \mathrm{t})=\operatorname{Re}\left[E_{\xi}(\xi, \eta) \mathrm{e}^{-\mathrm{i} \omega \mathrm{t}}\right]=\frac{2}{\varepsilon_{\mathrm{j}} \lambda_{0} k_{0} \sqrt{\xi+\eta}} \frac{\partial}{\partial \eta}\left[H_{\phi}\left(\xi, \eta, t-\frac{\pi}{2 \omega}\right) \sqrt{\eta}\right] \\
& E_{\eta}(\xi, \eta, \mathrm{t})=\operatorname{Re}\left[E_{\eta}(\xi, \eta) \mathrm{e}^{-\mathrm{i} \omega \mathrm{t}}\right]=-\frac{2}{\varepsilon_{\mathrm{j}} \lambda_{0} \mathrm{k}_{0} \sqrt{\xi+\eta}} \frac{\partial}{\partial \xi}\left[\mathrm{H}_{\phi}\left(\xi, \eta, t-\frac{\pi}{2 \omega}\right) \sqrt{\xi}\right] .
\end{aligned}
$$

By substituting (189) and (190) into (186), we obtain a differential equation expressed in terms of the total derivatives with respect to $\xi$ and $\eta$ as follows: 


$$
\frac{\partial}{\partial \xi}\left[\mathrm{H}_{\phi}\left(\xi, \eta, t-\frac{\pi}{2 \omega}\right) \sqrt{\xi}\right] \frac{\mathrm{d} \xi}{\sqrt{\xi}}+\frac{\partial}{\partial \eta}\left[H_{\phi}\left(\xi, \eta, t-\frac{\pi}{2 \omega}\right) \sqrt{\eta}\right] \frac{\mathrm{d} \eta}{\sqrt{\eta}}=0,
$$

which can be described by the exact differential form

$$
d \psi(\xi, \eta)=\frac{\partial}{\partial \xi} \psi(\xi, \eta) d \xi+\frac{\partial}{\partial \eta} \psi(\xi, \eta) d \eta=0
$$

with the solution

$$
\psi(\xi, \eta)=\text { constant }
$$

if we set

$$
\psi(\xi, \eta)=H_{\phi}\left(\xi, \eta, t-\frac{\pi}{2 \omega}\right) \sqrt{\xi \eta}
$$

Because the time-varying scalar field for the electric field-line representation, denoted by $f(\xi, \eta, t)$, is proportional to the sinusoidal time dependence $e^{-i \omega t}$, by taking into account (184) when estimating (194), we can set

$$
f(\xi, \eta, t)=H_{\phi}\left(\xi, \eta, t-\frac{\pi}{2 \omega}\right) \sqrt{\xi \eta} .
$$

The field-line pattern at time $t=t_{0}$ is described by the scalar field with the contour

$$
f\left(\xi, \eta, t_{0}\right)=C,
$$

where $\mathrm{C}$ indicates the contour level. The contour interval can be controlled by the interval value of the contour level. The evolution of field-line patterns can be investigated for different moments:

$$
t=t_{0}+n \tau \quad \text { with } \quad n=0,1,2,3, \ldots
$$

where $\tau$ denotes a suitable chosen duration between two neighbouring snapshots.

For actual calculations, we rewrite (195) in a form that contains the unified radial function and the extended angular function. By using (184), (4), (8) and (42) for (195), we obtain the scalar field for the electric field-line representation as follows:

$$
f(\xi, \eta, t)=\operatorname{Re}\left[\Xi_{u}(\xi) \mathrm{H}_{j}(\eta, \xi) \sqrt{\xi \eta} \exp (-\mathrm{i} \omega t+\mathrm{i} \pi / 2)\right], \quad j=1,2
$$

in which we actually have to use an approximate solution of the unified radial function and the extended angular function. The electric field-line representation becomes more accurate when a higher-order approximate solution is used. When the zeroth-order approximated solution is used in (198), the scalar field for the electric field-line representation becomes 


$$
f^{0 \text { th }}(\xi, \eta, t)=\operatorname{Re}\left[\Xi_{u}^{(0)}(\xi) \mathrm{H}_{j}^{(0)}(\eta, \xi) \sqrt{\xi \eta} \exp (-\mathrm{i} \omega t+\mathrm{i} \pi / 2)\right], \quad j=1,2
$$

The first-order approximated solution becomes

$$
f^{1 \mathrm{st}}(\xi, \eta, t)=\operatorname{Re}\left[\left\{\boldsymbol{\Xi}_{u}^{(0)}(\xi)+\Xi_{u}^{(1)}(\xi)\right\}\left\{\mathrm{H}_{j}^{(0)}(\eta, \xi)+\mathrm{H}_{j}^{(1)}(\eta, \xi)\right\} \sqrt{\xi \eta} \exp (-\mathrm{i} \omega t+\mathrm{i} \pi / 2)\right], j=1,2 .
$$

In the same manner, we can express the scalar field for the electric-field-line representation when the second-order approximated solution is provided.

Figure 6 shows the electric field-line patterns of the zeroth-order approximated plasmonic modes of the metallic hollow paraboloid for the surfaces (a) $\eta_{0}=0.3$ and (b) $\eta_{0}=0.6$, calculated by using (199) with $t=\pi / 2 \omega$. In the calculations, we employ $\mathrm{H}_{1}^{(0)}(\eta, \xi), \mathrm{H}_{2}^{(0)}(\eta, \xi)$, and $\Xi_{u}^{(0)}(\xi)$ in (96), (109), and (143), respectively, with $\varepsilon_{d}=1$, $\varepsilon_{m}=-20, s=\lambda_{0}, H_{0}^{\prime \prime}=1$, and some specific values in table 1 . The geometrical dimensions in figure 6 are normalized by the wavelength in vacuum, $\lambda_{0}$, on the horizontal and vertical axes. The blue and red lines in figure 6 indicate clockwise and counterclockwise loops, respectively, for the line of electric force. The field-patterns in figure 6 evolve with the behaviour of the standing waves. We select the two values of the parameter $\eta_{0}\left(=\rho_{0} / \lambda_{0}\right)$ in figure 6 to show the contrast between the positive and negative values of the Sommerfeld parameter for the irregular Coulomb wave function $G_{0}$ in the zeroth-order unified radial function (143). Parts (a) and (b) in figure 6 correspond to positive and negative values of the Sommerfeld parameter $\gamma$, respectively, because of the values of (a) $\gamma=0.3464$ and (b) $\gamma=-0.3295$, calculated on the basis of $\gamma=-\zeta_{u}^{(0)}(0) / \lambda_{0} k_{m p}(0)$ in (130) by using the specific values of $\zeta_{u}^{(0)}(0)$ for (a) $\eta_{0}=0.3$ and (b) $\eta_{0}=0.6$ in table 1 . The apparent distinction between parts (a) and (b) in figure 6 can be found in the behaviour of the electric field-line pattern along the vertical line passing through the paraboloid apex: the vertical electric field through the paraboloid apex is stronger for part (a) as compared to that for part (b), particularly around the origin of figures. This distinction can be understood from the viewpoint of the positive and negative values of the Sommerfeld parameter $\gamma$ for the irregular Coulomb wave function $G_{0}$ in (143); from (B.11) and (139), it is inferred that

$$
G_{0}\left(\gamma, \frac{\lambda_{0} k_{m p}(0)}{2} \xi\right) \sim \frac{1}{C_{0}(\gamma)}=\sqrt{\frac{\mathrm{e}^{2 \pi \gamma}-1}{2 \pi \gamma}} \quad(\xi \rightarrow 0)
$$


which results in (a) $G_{0}(\gamma, 0)=1.895$ at $\gamma=0.3464$ for $\eta_{0}=0.3$, and (b) $G_{0}(\gamma, 0)=0.6382$ at $\gamma=-0.3295$ for $\eta_{0}=0.6$; for reference, $G_{0}(\gamma, 0)=1$ at $\gamma=0$. Equation (201) indicates that $G_{0}(\gamma, 0)$ rapidly increases as a function of the positive value of the Sommerfeld parameter $\gamma$, whereas $G_{0}(\gamma, 0)$ gradually increases as a function of the negative value of $\gamma$; this implies that the zeroth-order plasmonic modes more strongly localize at $\xi=0$ or the vertical line through the paraboloidal apex with an increasing positive value of $\gamma$. The Sommerfeld parameter $\gamma$ is closely related to (96) in the dielectric region for $\xi=0$ :

$$
\mathrm{H}_{1}^{(0)}(\eta, 0)=\sqrt{\eta_{0} / \eta} F_{0}\left(\kappa_{d}(0), \eta \pi \varepsilon_{d}^{1 / 2}\right) / F_{0}\left(\kappa_{d}(0), \eta_{0} \pi \varepsilon_{d}^{1 / 2}\right), \quad 0 \leq \eta \leq \eta_{0}
$$

with

$$
\kappa_{d}(0)=\frac{\zeta_{u}^{(0)}(0)}{2 \pi \varepsilon_{d}{ }^{1 / 2}}
$$

where we employ (111) and (88). Equation (203) shows that the Sommerfeld parameter $\kappa_{d}(0)$ for the regular Coulomb wave function $F_{0}$ in the zeroth-order angular function (202) in the dielectric region is opposite in sign to the Sommerfeld parameter $\gamma$ defined as $\gamma=-\zeta_{u}^{(0)}(0) / \lambda_{0} k_{m p}(0)$ in (130). From (B.10) and (95), it is inferred that

$$
F_{0}\left(\kappa_{d}(0), \eta \pi \varepsilon_{d}^{1 / 2}\right) \sim \eta \pi \varepsilon_{d}^{1 / 2} C_{0}\left(\kappa_{d}(0)\right)=\eta \pi \varepsilon_{d}^{1 / 2} \sqrt{\frac{2 \pi \kappa_{d}(0)}{e^{2 \pi \kappa_{d}(0)}-1}} \quad(\eta \rightarrow 0) .
$$

By using (204) for (202), we obtain $\mathrm{H}_{1}^{(0)}(\eta, 0) \sim 3.154 \sqrt{\eta}$ as $\eta \rightarrow 0$ for $\eta_{0}=0.3$ (a) and $\mathrm{H}_{1}^{(0)}(\eta, 0) \sim 1.232 \sqrt{\eta}$ as $\eta \rightarrow 0$ for $\eta_{0}=0.6$ (b). Equation (204) indicates that the value of $\mathrm{H}_{1}^{(0)}(\eta, 0) / \sqrt{\eta}$ for $\eta \rightarrow 0$ rapidly decreases as a function of the negative value of the Sommerfeld parameter $\kappa_{d}(0)$, whereas it slowly decrease as a function of the positive value of $\kappa_{d}(0)$; this implies that the zeroth-order plasmonic modes for the metallic hollow paraboloid much more strongly localize around the paraboloid apex in the dielectric region with an decreasing negative value of $\kappa_{d}(0)$, or equally with an increasing positive value of $\gamma$ because of (130) and (203). Hence, the stronger electric field around the origin of the figures for part (a) as compared to that for part (b) can be attributed to the following two factors: the rapidly increasing values of the zeroth-order unified radial function (143) as $\xi \rightarrow 0$ and the stronger localization in the dielectric region of the zeroth-order angular function (96) around the paraboloidal apex.

Figure 7 shows the electric field-line pattern of the zeroth-order approximated 
plasmonic modes of the metallic hollow paraboloid for the surface $\eta_{0}=0.429976$; the specific value of $\eta_{0}$ for $\zeta_{u}^{(0)}(0)=0$ is calculated from (116) under the conditions $\varepsilon_{m}=-20$ and $\varepsilon_{d}=1$. The other calculation conditions for drawing figure 7 are identical to those in figure 6 . Because $\zeta_{u}^{(0)}(0)=0$, we obtain $\gamma=0, \kappa_{d}(0)=0$ and $\kappa_{m}(0)=0$ from (130), (203), and (100), respectively. By substituting $\gamma=0$ into (143), we simplify the unified radial function of the zeroth order into the form

$$
\Xi_{u}^{(0)}(\xi)=2 H_{0}^{\prime \prime} \xi^{-1 / 2} \cos \left(\frac{\lambda_{0} k_{m p}(0)}{2} \xi\right)
$$

where we use the following formula (see equation 14.6.4 of [53])

$$
G_{0}(0, \rho)=\cos \rho \text {. }
$$

By substituting $\kappa_{d}(0)=0$ into (202), and by using (114), we obtain

$$
\mathrm{H}_{1}^{(0)}(\eta, 0)=\sqrt{\eta_{0} / \eta} \sin \left(\eta \pi \varepsilon_{d}^{1 / 2}\right) / \sin \left(\eta_{0} \pi \varepsilon_{d}^{1 / 2}\right), \quad 0 \leq \eta \leq \eta_{0} .
$$

By substituting $\kappa_{m}(0)=0$ into (109) in the metallic region for $\xi=0$, and then by using (115), we obtain

$$
\mathrm{H}_{2}^{(0)}(\eta, 0)=\sqrt{\eta_{0} / \eta} \exp \left[-\left(\eta-\eta_{0}\right) \pi\left|\varepsilon_{m}\right|^{1 / 2}\right], \quad \eta_{0} \leq \eta<\infty .
$$

Because equations (205), (207), and (208) are described by simple functions such as trigonometric and exponential functions, we can thoroughly study the electric field-line pattern of figure 7 , calculated by using (199) with $t=\pi / 2 \omega$, as compared to that of figure 6. For $\xi=0$ and $t=\pi / 2 \omega$, the zeroth-order scalar field for the electric field-line representation in (199) is described from (205), (207) and (208) as

$$
f^{0 \text { th }}(0, \eta, \pi / 2 \omega)=2 H_{0}^{\prime \prime} \sqrt{\eta_{0}} \times \begin{cases}\sin \left(\eta \pi \varepsilon_{d}{ }^{1 / 2}\right) / \sin \left(\eta_{0} \pi \varepsilon_{d}{ }^{1 / 2}\right), & 0 \leq \eta \leq \eta_{0} \\ \exp \left[-\left(\eta-\eta_{0}\right) \pi\left|\varepsilon_{m}\right|^{1 / 2}\right], & \eta_{0} \leq \eta<\infty\end{cases}
$$

which directly corresponds to the behaviour of the electric field-line pattern along the vertical line passing through the paraboloid apex in figure 7. Because the electric field-line pattern of figure 7 corresponds to the case $\gamma=0$, it is used as a fixed standard to thoroughly understand the electric field-line pattern of parts (a) and (b) in figure 6 with $\gamma>0$ and $\gamma<0$, respectively.

In this study, we do not show the electric field-line patterns of the first-order approximated plasmonic modes of the metallic hollow paraboloid by using (200) because of some difficulties involved in numerical calculations. One of these difficulties 
lies in the impossibility of writing the first-order unified separation quantity (171) with elementary functions without any integrals. Another difficulty lies in the impossibility of numerically calculating the first-order unified separation quantity (171) for unrestricted values of $\xi$ by usual numerical methods for computing special functions such as those currently used in commercial computer programs such as Mathematica. Other difficulties lie in employing numerical calculus for double integration; it is a time-consuming and complex job for our present aim of identifying plasmonic modes in circular paraboloidal geometry. 


\section{Conclusions}

We studied plasmonic modes for circular paraboloidal geometry by solving the wave equation for the magnetic field in paraboloidal coordinates through the QSOV in combination with perturbation methods. By analytically solving the zeroth-order perturbation equations for unified radial and extended angular functions, we found that plasmonic modes are absent for the metallic solid paraboloid but present for the metallic hollow paraboloid in the form of standing waves and not as superfocusing waves. For the metallic hollow paraboloid, we showed that the unified radial function of the zeroth order is described by using the irregular Coulomb wave functions, and that the extended angular functions of the zeroth order are described by using the regular Coulomb wave functions and the Whittaker functions of the second kind in the dielectric and metallic regions, respectively. The unified radial and extended angular functions of the first order were obtained as analytic forms, including definite integrals, but they were not estimated numerically because of some difficulties involved in numerical calculations. By using the zeroth-order perturbed solutions, we obtained the electric field-line patterns of the zeroth-order approximated plasmonic modes in the metallic hollow paraboloid for relatively small and large radii of curvature at the paraboloid apex. The electric field-line patterns showed that the electric field around the paraboloid apex is stronger for the small radius of curvature as compared to that for the large one; this result was analytically studied from the mathematical viewpoint of the zeroth-order unified radial function and the extended angular function in the dielectric region. We firmly believe that the QSOV method employed for solving the wave equation is the optimum method to theoretically understand plasmonic modes in various tapered geometries. 


\section{Acknowledgments}

The authors are most thankful to Dr. Y. Katagiri and Dr. K. Kasai of NICT for their useful discussions and comments. 
Appendix A. Differential equations for the azimuthal magnetic field of transverse magnetic waves in a parabolic system of coordinates

For the magnetic field $\boldsymbol{H}(\boldsymbol{x}, t)$ at time $t$ at the point located by the coordinate vector $\boldsymbol{x}$, we assume a time dependence of $\mathrm{e}^{-\mathrm{i} \omega \mathrm{t}}$ and therefore describe

$$
\boldsymbol{H}(\boldsymbol{x}, t)=\operatorname{Re}[\boldsymbol{H}(\boldsymbol{x}) \exp (-\mathrm{i} \omega \mathrm{t})] .
$$

By using the two Maxwell curl equations in the absence of sources, for the magnetic field in (A.1) we obtain

$$
\nabla \times(\nabla \times \boldsymbol{H}(\boldsymbol{x}))-\varepsilon \frac{\omega^{2}}{\mathrm{c}^{2}} \boldsymbol{H}(\boldsymbol{x})=0 .
$$

In the paraboloidal system of coordinates, we express the magnetic field $\boldsymbol{H}(\boldsymbol{x})$ of transverse magnetic (TM) waves as

$$
\boldsymbol{H}(\boldsymbol{x})=\left(H_{\xi}, H_{\eta}, H_{\phi}\right)
$$

where

$$
H_{\xi}=0, \quad H_{\eta}=0, \quad H_{\phi}=H_{\phi}(\xi, \eta, \phi) .
$$

The metric coefficients of the paraboloidal coordinates in (1) are

$$
\mathrm{h}_{\xi}=\frac{\mathrm{s}}{2} \sqrt{\frac{\eta}{\xi}+1}, \quad \mathrm{~h}_{\eta}=\frac{\mathrm{s}}{2} \sqrt{\frac{\xi}{\eta}+1}, \quad \mathrm{~h}_{\phi}=\mathrm{s} \sqrt{\xi \eta} .
$$

By using (A.3)-(A.5), the curl of $\boldsymbol{H}(\boldsymbol{x})$ is obtained as

$$
\begin{gathered}
(\nabla \times \boldsymbol{H}(\boldsymbol{x}))_{\xi}=\frac{1}{\mathrm{~h}_{\eta} \mathrm{h}_{\phi}}\left[\frac{\partial}{\partial \eta}\left(H_{\phi} \mathrm{h}_{\phi}\right)-\frac{\partial}{\partial \phi}\left(H_{\eta} \mathrm{h}_{\eta}\right)\right]=\frac{1}{\mathrm{~h}_{\eta} \mathrm{h}_{\phi}} \frac{\partial}{\partial \eta}\left(H_{\phi} \mathrm{h}_{\phi}\right) \\
(\nabla \times \boldsymbol{H}(\boldsymbol{x}))_{\eta}=\frac{1}{\mathrm{~h}_{\phi} \mathrm{h}_{\xi}}\left[\frac{\partial}{\partial \phi}\left(H_{\xi} \mathrm{h}_{\xi}\right)-\frac{\partial}{\partial \xi}\left(H_{\phi} \mathrm{h}_{\phi}\right)\right]=-\frac{1}{\mathrm{~h}_{\phi} \mathrm{h}_{\xi}} \frac{\partial}{\partial \xi}\left(H_{\phi} \mathrm{h}_{\phi}\right) \\
(\nabla \times \boldsymbol{H}(\boldsymbol{x}))_{\phi}=\frac{1}{\mathrm{~h}_{\xi} \mathrm{h}_{\eta}}\left[\frac{\partial}{\partial \xi}\left(H_{\eta} \mathrm{h}_{\eta}\right)-\frac{\partial}{\partial \eta}\left(H_{\xi} \mathrm{h}_{\xi}\right)\right]=0 .
\end{gathered}
$$

By using (A.3)-(A.8), the curl of the curl of $\boldsymbol{H}(\boldsymbol{x})$ equals

$$
\begin{aligned}
\{\nabla \times(\nabla \times \boldsymbol{H}(\boldsymbol{x}))\}_{\xi} & =\frac{1}{\mathrm{~h}_{\eta} \mathrm{h}_{\phi}}\left[\frac{\partial}{\partial \eta}\left((\nabla \times \boldsymbol{H}(\boldsymbol{x}))_{\phi} \mathrm{h}_{\phi}\right)-\frac{\partial}{\partial \phi}\left((\nabla \times \boldsymbol{H}(\boldsymbol{x}))_{\eta} \mathrm{h}_{\eta}\right)\right] \\
& =\frac{1}{\mathrm{~h}_{\eta} \mathrm{h}_{\phi}} \frac{\partial}{\partial \phi}\left(\frac{\mathrm{h}_{\eta}}{\mathrm{h}_{\phi} \mathrm{h}_{\xi}} \frac{\partial}{\partial \xi}\left(H_{\phi} \mathrm{h}_{\phi}\right)\right)=\frac{2}{\mathrm{~s}^{2} \sqrt{\eta(\xi+\eta)}} \frac{\partial^{2}}{\partial \phi \partial \xi}\left[H_{\phi}(\xi, \eta, \phi) \sqrt{\xi}\right]
\end{aligned}
$$




$$
\begin{aligned}
\{\nabla \times(\nabla \times \boldsymbol{H}(\boldsymbol{x}))\}_{\eta}= & \frac{1}{\mathrm{~h}_{\phi} \mathrm{h}_{\xi}}\left[\frac{\partial}{\partial \phi}\left((\nabla \times \boldsymbol{H}(\boldsymbol{x}))_{\xi} \mathrm{h}_{\xi}\right)-\frac{\partial}{\partial \xi}\left((\nabla \times \boldsymbol{H}(\boldsymbol{x}))_{\phi} \mathrm{h}_{\phi}\right)\right] \\
= & \frac{1}{\mathrm{~h}_{\phi} \mathrm{h}_{\xi}} \frac{\partial}{\partial \phi}\left(\frac{\mathrm{h}_{\xi}}{\mathrm{h}_{\eta} \mathrm{h}_{\phi}} \frac{\partial}{\partial \eta}\left(H_{\phi} \mathrm{h}_{\phi}\right)\right)=\frac{2}{\mathrm{~s}^{2} \sqrt{\xi(\xi+\eta)}} \frac{\partial^{2}}{\partial \phi \partial \eta}\left[H_{\phi}(\xi, \eta, \phi) \sqrt{\eta}\right] \\
\{\nabla \times(\nabla \times \boldsymbol{H}(\boldsymbol{x}))\}_{\phi} & =\frac{1}{\mathrm{~h}_{\xi} \mathrm{h}_{\eta}}\left[\frac{\partial}{\partial \xi}\left((\nabla \times \boldsymbol{H}(\boldsymbol{x}))_{\eta} \mathrm{h}_{\eta}\right)-\frac{\partial}{\partial \eta}\left((\nabla \times \boldsymbol{H}(\boldsymbol{x}))_{\xi} \mathrm{h}_{\xi}\right)\right] \\
& =\frac{1}{\mathrm{~h}_{\xi} \mathrm{h}_{\eta}}\left[\frac{\partial}{\partial \xi}\left(-\frac{\mathrm{h}_{\eta}}{\mathrm{h}_{\phi} \mathrm{h}_{\xi}} \frac{\partial}{\partial \xi}\left(H_{\phi} \mathrm{h}_{\phi}\right)\right)-\frac{\partial}{\partial \eta}\left(\frac{\mathrm{h}_{\xi}}{\mathrm{h}_{\eta} \mathrm{h}_{\phi}} \frac{\partial}{\partial \eta}\left(H_{\phi} \mathrm{h}_{\phi}\right)\right)\right] \\
& =-\frac{4}{\mathrm{~s}^{2}} \frac{\sqrt{\xi}}{\xi+\eta} \frac{\partial^{2}}{\partial \xi^{2}}\left[H_{\phi}(\xi, \eta, \phi) \sqrt{\xi}\right]-\frac{4}{\mathrm{~s}^{2}} \frac{\sqrt{\eta}}{\xi+\eta} \frac{\partial^{2}}{\partial \eta^{2}}\left[H_{\phi}(\xi, \eta, \phi) \sqrt{\eta}\right] .
\end{aligned}
$$

By substituting (A.9)-(A.11) into (A.2), we obtain

$$
\begin{gathered}
\frac{\partial^{2}}{\partial \phi \partial \xi}\left[H_{\phi}(\xi, \eta, \phi) \sqrt{\xi}\right]=0 \\
\frac{\partial^{2}}{\partial \phi \partial \eta}\left[H_{\phi}(\xi, \eta, \phi) \sqrt{\eta}\right]=0 \\
\frac{4}{\mathrm{~s}^{2}} \frac{\sqrt{\xi}}{\xi+\eta} \frac{\partial^{2}}{\partial \xi^{2}}\left[H_{\phi}(\xi, \eta, \phi) \sqrt{\xi}\right]+\frac{4}{\mathrm{~s}^{2}} \frac{\sqrt{\eta}}{\xi+\eta} \frac{\partial^{2}}{\partial \eta^{2}}\left[H_{\phi}(\xi, \eta, \phi) \sqrt{\eta}\right]+\varepsilon \frac{\omega^{2}}{\mathrm{c}^{2}} H_{\phi}(\xi, \eta, \phi)=0
\end{gathered}
$$

By solving the partial differential equations in (A.12) and (A.13), we obtain

$$
\begin{aligned}
& H_{\phi}(\xi, \eta, \phi) \sqrt{\xi}=\mathrm{f}_{1}(\xi, \eta)+\mathrm{g}_{1}(\phi, \eta) \\
& H_{\phi}(\xi, \eta, \phi) \sqrt{\eta}=\mathrm{f}_{2}(\xi, \eta)+\mathrm{g}_{2}(\phi, \xi),
\end{aligned}
$$

respectively, where $\mathrm{f}_{1}(\xi, \eta), \mathrm{f}_{2}(\xi, \eta), \mathrm{g}_{1}(\phi, \eta)$, and $\mathrm{g}_{2}(\phi, \xi)$ are arbitrary functions. Rewriting (A.15) and (A.16) as

$$
H_{\phi}(\xi, \eta, \phi)=\frac{\mathrm{f}_{1}(\xi, \eta)}{\sqrt{\xi}}+\frac{\mathrm{g}_{1}(\phi, \eta)}{\sqrt{\xi}}
$$




$$
H_{\phi}(\xi, \eta, \phi)=\frac{\mathrm{f}_{2}(\xi, \eta)}{\sqrt{\eta}}+\frac{\mathrm{g}_{2}(\phi, \xi)}{\sqrt{\eta}}
$$

respectively and by comparing the equations, we obtain

$$
H_{\phi}(\xi, \eta, \phi)=\mathrm{f}(\xi, \eta)+\frac{\mathrm{g}(\phi)}{\sqrt{\xi \eta}}
$$

where $\mathrm{f}(\xi, \eta)$ and $\mathrm{g}(\phi)$ are arbitrary functions and satisfy the following relations:

$$
\begin{aligned}
& \mathrm{f}(\xi, \eta)=\frac{\mathrm{f}_{1}(\xi, \eta)}{\sqrt{\xi}}=\frac{\mathrm{f}_{2}(\xi, \eta)}{\sqrt{\eta}} \\
& \frac{\mathrm{g}(\phi)}{\sqrt{\xi \eta}}=\frac{\mathrm{g}_{1}(\phi, \eta)}{\sqrt{\xi}}=\frac{\mathrm{g}_{2}(\phi, \xi)}{\sqrt{\eta}} .
\end{aligned}
$$

On the right-hand side of (A.19), the first term, $\mathrm{f}(\xi, \eta)$, is distinguished from the second term, $\mathrm{g}(\phi) / \sqrt{\xi \eta}$, by the type of solutions obtained through the (quasi-)separation of variables. Hence, we divide solutions of $H_{\phi}(\xi, \eta, \phi)$ into two types, as follows:

$$
\begin{array}{ll}
H_{\phi}(\xi, \eta, \phi)=\mathrm{f}(\xi, \eta) & (\text { type I) } \\
H_{\phi}(\xi, \eta, \phi)=\frac{\mathrm{g}(\phi)}{\sqrt{\xi \eta}} \quad(\text { type II }) .
\end{array}
$$

The solutions of type II in (A.23) are almost worthless because they do not satisfy the differential equation in (A.14) for any of the values of $\varepsilon$, except for $\varepsilon=0$. It is worth examining the solutions of type I in (A.22), which is independent of the azimuthal angle $\phi$ and therefore allows us to describe the differential equation in (A.14) in the simplified form

$$
\frac{4}{\mathrm{~s}^{2}} \frac{\sqrt{\xi}}{\xi+\eta} \frac{\partial^{2}}{\partial \xi^{2}}\left[H_{\phi}(\xi, \eta) \sqrt{\xi}\right]+\frac{4}{\mathrm{~s}^{2}} \frac{\sqrt{\eta}}{\xi+\eta} \frac{\partial^{2}}{\partial \eta^{2}}\left[H_{\phi}(\xi, \eta) \sqrt{\eta}\right]+\varepsilon \frac{\omega^{2}}{\mathrm{c}^{2}} H_{\phi}(\xi, \eta)=0
$$

This is a differential equation for the azimuthal magnetic field of TM waves and is actually solved with the paraboloidal system of coordinates. 


\section{Appendix B. Some properties of Coulomb wave functions}

Coulomb wave functions are one of the most basic objects of particle theory in quantum physics; therefore, their descriptions can be found everywhere [52-54, 60-63]. We briefly describe the standard Coulomb wave functions $F_{L}(\eta, \rho)$ and $G_{L}(\eta, \rho)$, which are solutions of the differential equation:

$$
\frac{\mathrm{d}^{2} w}{\mathrm{~d} \rho^{2}}+\left[1-\frac{2 \eta}{\rho}-\frac{L(L+1)}{\rho^{2}}\right] w=0
$$

where $L$ denotes the orbital angular momentum and $\eta$ denotes the Sommerfeld parameter $[52,63]$.

The Coulomb wave functions can be expressed with confluent hypergeometric functions. The regular Coulomb wave function $F_{L}(\eta, \rho)$ can be expressed as [53]

$$
\begin{gathered}
F_{L}(\eta, \rho)=C_{L}(\eta) \rho^{\mathrm{L}+1} \mathrm{e}^{-\mathrm{i} \rho} M(L+1-\mathrm{i} \eta, 2 L+2,2 \mathrm{i} \rho) \\
C_{L}(\eta)=2^{L} \mathrm{e}^{-\pi \eta / 2} \frac{|\Gamma(L+1+\mathrm{i} \eta)|}{\Gamma(2 L+2)}
\end{gathered}
$$

where the $M$-function is the confluent hypergeometric function of the first kind, often denoted by ${ }_{1} F_{1}$. For non-negative integer $L, C_{L}(\eta)$ in (B.3) can be simplified into the following expression [62]:

$$
C_{L}(\eta)=\frac{2^{L}}{(2 L+1) !}\left[\frac{2 \pi \eta}{\mathrm{e}^{2 \pi \eta}-1} \prod_{p=0}^{L}\left(\eta^{2}+p^{2}\right)\right]^{1 / 2} .
$$

The irregular Coulomb wave function $G_{L}(\eta, \rho)$ can be expressed as [61]

$$
\begin{gathered}
G_{L}(\eta, \rho)=\mathrm{e}^{\pi \eta / 2} \mathrm{e}^{\mathrm{i}\left\{\sigma_{\mathrm{L}}(\eta)-L \pi / 2\right\}} W_{-\mathrm{i} \eta, \mathrm{L}+1 / 2}(-2 \mathrm{i} \rho)-\mathrm{i} F_{L}(\eta, \rho) \\
\sigma_{L}(\eta)=\arg \Gamma(L+1+\mathrm{i} \eta),
\end{gathered}
$$

where $W_{-i \eta, L+1 / 2}(-2 i \rho)$ represents the Whittaker function of the second kind, defined by [55]:

$$
W_{\kappa, \mu}(z)=\mathrm{e}^{-z / 2} z^{1 / 2+\mu} U\left(\frac{1}{2}+\mu-\kappa, 2 \mu+1, z\right) .
$$

Here the $U$-function is the confluent hypergeometric function of the second kind. Incidentally, the Whittaker function of the first kind is expressed by [55]

$$
M_{\kappa, \mu}(z)=\mathrm{e}^{-z / 2} z^{1 / 2+\mu} M\left(\frac{1}{2}+\mu-\kappa, 2 \mu+1, z\right),
$$


and the Wroskian relation is [45]:

$$
M_{\kappa, \mu}(z) \frac{\mathrm{d}}{\mathrm{d} z} \mathrm{~W}_{\kappa, \mu}(z)-\mathrm{W}_{\kappa, \mu}(z) \frac{\mathrm{d}}{\mathrm{d} z} M_{\kappa, \mu}(z)=-\frac{\Gamma(2 \mu+1)}{\Gamma\left(\frac{1}{2}-\kappa+\mu\right)}, \quad 2 \mu+1 \neq 0,-1,-2, \ldots .
$$

The functions $F_{L}(\eta, \rho)$ and $G_{L}(\eta, \rho)$ are real for real values of $\eta, \rho>0$, $L \geq 0$ [52]. For the limit of $\rho \rightarrow 0$, they behave as [60]:

$$
\begin{gathered}
F_{L}(\eta, \rho) \sim C_{L}(\eta) \rho^{L+1}\left(1+\frac{\eta}{\mathrm{L}+1} \rho+\cdots\right) \quad(\rho \rightarrow 0) \\
G_{L}(\eta, \rho) \sim \frac{1}{(2 L+1) C_{L}(\eta)} \rho^{-L}\left[1+\left(\begin{array}{ccc}
O(\eta \rho \ln \rho) & \text { if } & \mathrm{L}=0 \\
O\left(\frac{\eta}{\mathrm{L}} \rho\right) & \text { if } & \mathrm{L} \neq 0
\end{array}\right)\right]
\end{gathered} \quad(\rho \rightarrow 0) .
$$

For the limit of $\rho \rightarrow \infty$, they behave as $[60,62]$ :

$$
\begin{array}{ll}
F_{L}(\eta, \rho) \sim \sin \left[\rho-\eta \ln (2 \rho)-\frac{L}{2} \pi+\sigma_{L}(\eta)\right] & (\rho \rightarrow \infty) \\
G_{L}(\eta, \rho) \sim \cos \left[\rho-\eta \ln (2 \rho)-\frac{L}{2} \pi+\sigma_{L}(\eta)\right] & (\rho \rightarrow \infty) .
\end{array}
$$

Their Wronskian relation is $[60,62]$ :

$$
G_{L}(\eta, \rho) \frac{d}{d \rho} F_{L}(\eta, \rho)-F_{L}(\eta, \rho) \frac{d}{d \rho} G_{L}(\eta, \rho)=1 .
$$

From equation 14.6.9 in [53], we obtain useful asymptotic formulas

$$
\begin{array}{ll}
F_{0}(\eta, \rho) \sim \frac{1}{2}\left(\frac{\rho}{2 \eta}\right)^{1 / 4} \exp (2 \sqrt{2 \eta \rho}-\pi \eta) & (2 \eta>>\rho) \\
\frac{d}{d \rho} F_{0}(\eta, \rho) \sim \frac{1}{2}\left(\frac{\rho}{2 \eta}\right)^{-1 / 4} \exp (2 \sqrt{2 \eta \rho}-\pi \eta) & (2 \eta>>\rho),
\end{array}
$$

which are used in appendix $C$.

Outgoing and incoming Coulomb wave functions [63] are defined as

$$
H_{L}^{+}(\eta, \rho)=G_{L}(\eta, \rho)+i F_{L}(\eta, \rho)
$$

and

$$
H_{L}^{-}(\eta, \rho)=G_{L}(\eta, \rho)-i F_{L}(\eta, \rho),
$$

respectively. For the limit of $\rho \rightarrow \infty$, they behave as 


$$
\begin{aligned}
& H_{L}^{+}(\eta, \rho) \sim \exp \left[i\left(\rho-\eta \ln (2 \rho)-\frac{L}{2} \pi+\sigma_{L}(\eta)\right)\right] \quad(\rho \rightarrow \infty) \\
& H_{L}^{-}(\eta, \rho) \sim \exp \left[-i\left(\rho-\eta \ln (2 \rho)-\frac{L}{2} \pi+\sigma_{L}(\eta)\right)\right] \quad(\rho \rightarrow \infty),
\end{aligned}
$$

which are easily derived from (B.12) and (B.13). For the limit of $\rho \rightarrow 0$, they behave for $L \geq 0$ as

$$
\begin{array}{cc}
H_{L}^{+}(\eta, \rho) \sim \frac{1}{(2 L+1) C_{L}(\eta)} \rho^{-L} & (\rho \rightarrow 0, \mathrm{~L} \geq 0) \\
H_{L}^{-}(\eta, \rho) \sim \frac{1}{(2 L+1) C_{L}(\eta)} \rho^{-L} & (\rho \rightarrow 0, \mathrm{~L} \geq 0),
\end{array}
$$

which are easily derived from (B.10) and (B.11). 


\section{Appendix C. Approximate behaviour of $\zeta_{u}^{(0)}(\xi)$ in (110) for the limit of $\xi \rightarrow \infty$}

Because it is difficult to solve (110) numerically for large $\xi$, it is very important to know the approximate behaviour of $\zeta_{u}^{(0)}(\xi)$ for the limit of $\xi \rightarrow \infty$.

From (B.15) and (B.16), it is inferred that

$$
\frac{F_{0}^{\prime}(\eta, \rho)}{F_{0}(\eta, \rho)} \sim \sqrt{\frac{2 \eta}{\rho}} \quad(2 \eta>>\rho),
$$

where $F_{L}^{\prime}(\eta, \rho)=\partial F_{L}(\eta, \rho) / \partial \rho$. According to (20) of section 7 [46] and (4.4.35) of chapter 4 [54], we see that

$$
W_{-\kappa, \mu}(z) \sim 2^{-1 / 2} \mathrm{z}^{1 / 4} \kappa^{-1 / 4} \kappa^{-\kappa} \mathrm{e}^{\kappa} \mathrm{e}^{-2 \sqrt{\kappa z}} \quad(\kappa \rightarrow \infty),
$$

and then

$$
W_{-\kappa, \mu}^{\prime}(z) \sim 2^{-1 / 2} \mathrm{z}^{1 / 4} \kappa^{-1 / 4} \kappa^{-\kappa} \mathrm{e}^{\kappa} \mathrm{e}^{-2 \sqrt{\kappa z}}\left(-\sqrt{\frac{\kappa}{\mathrm{z}}}\right) \quad(\kappa \rightarrow \infty),
$$

where $W_{-\kappa, \mu}^{\prime}(z)=\partial W_{-\kappa, \mu}(z) / \partial z$. From (C.2) and (C.3), we see that

$$
\frac{W_{-\kappa, \mu}^{\prime}(z)}{W_{-\kappa, \mu}(z)} \sim-\sqrt{\frac{\kappa}{z}} \quad(\kappa \rightarrow \infty) .
$$

By using (C.1) and (C.4) for (110) with the limit $\xi \rightarrow \infty$, we obtain

$$
\frac{1}{2 \sqrt{\varepsilon_{d}}} \sqrt{\frac{2 \kappa_{d}(\xi)}{\frac{\eta_{0}}{2} s k_{0} \varepsilon_{d}^{1 / 2}}}=-\frac{1}{\sqrt{\left|\varepsilon_{m}\right|}}\left(-\sqrt{\frac{-\kappa_{m}(\xi)}{\eta_{0} s k_{0}\left|\varepsilon_{m}\right|^{1 / 2}}}\right) \quad(\xi \rightarrow \infty)
$$

or

$$
\frac{\kappa_{d}(\xi)}{\varepsilon_{d}^{3 / 2}}+\frac{\kappa_{m}(\xi)}{\left|\varepsilon_{m}\right|^{3 / 2}}=0 \quad(\xi \rightarrow \infty)
$$

By substituting (88) and (100) into (C.6), we obtain

$$
\frac{1}{\varepsilon_{d}^{2}}\left(\zeta_{u}^{(0)}(\xi)+\frac{s^{2} \beta_{1}^{2}}{4} \xi\right)-\frac{1}{\left|\varepsilon_{m}\right|^{2}}\left(\zeta_{u}^{(0)}(\xi)+\frac{s^{2} \beta_{2}^{2}}{4} \xi\right)=0 \quad(\xi \rightarrow \infty)
$$

or

$$
\left(\frac{1}{\varepsilon_{d}^{2}}-\frac{1}{\left|\varepsilon_{m}\right|^{2}}\right) \zeta_{u}^{(0)}(\xi)+\frac{s^{2}}{4}\left(\frac{\beta_{1}^{2}}{\varepsilon_{d}{ }^{2}}-\frac{\beta_{2}^{2}}{\left|\varepsilon_{m}\right|^{2}}\right) \xi=0 \quad(\xi \rightarrow \infty),
$$

which implies that $\zeta_{u}^{(0)}(\xi)$ is proportional to $\xi$ for $\xi \rightarrow \infty$. For the metallic hollow paraboloid, from (34), it is inferred that 


$$
\beta_{1}^{2}=k_{p}^{2}-\varepsilon_{d} k_{0}^{2}, \quad \beta_{2}^{2}=k_{p}^{2}-\varepsilon_{m} k_{0}^{2} .
$$

By substituting (C.9) into (C.8), and by using (7) and (27), we obtain

$$
\zeta_{u}^{(0)}(\xi) \sim 0 \cdot \xi \quad(\xi \rightarrow \infty) .
$$

This equation indicates that $\zeta_{u}^{(0)}(\xi)$ behaves as a straight line with a slope of zero for $\xi \rightarrow \infty$. 


\section{References}

[1] Raether H 1988 Surface Plasmons on Smooth and Rough Surfaces and on Gratings (Berlin: Springer)

[2] Maier S A, Brongersma M L, Kik P G, Meltzer S, Requicha A A G, Koel B E and Atwater H A 2001 Plasmonics - a route to nanoscale optical devices Adv. Mater. 13 1501

[3] Barnes W L, Dereux A and Ebbesen T W 2003 Surface plasmon subwavelength optics Nature $\mathbf{4 2 4} 824$

[4] Maier S A and Atwater H A 2005 Plasmonics: localization and guiding of electromagnetic energy in metal/dielectric structures J. Appl. Phys. 98011101

[5] Ozbay E 2006 Plasmonics: merging photonics and electronics at nanoscale dimensions Science 311189

[6] Oulton R F, Bartal G, Pile D F P and Zhang X 2008 Confinement and propagation characteristics of subwavelength plasmonic modes New J. Phys. 10105018

[7] Takahara J and Kobayashi T 2006 Low-dimensional optical waveguides and wavenumber surface Nanoplasmonics from Fundamentals to Applications (Handai Nanophotonics vol 2) ed S Kawata and H Masuhara (Amsterdam: Elsevier) p 55

[8] Wang C-J and Lin L Y 2007 Nanoscale waveguiding methods Nanoscale Res. Lett. 2219

[9] Maier S A 2008 Waveguiding - The best of both worlds Nature Photon. 2460

[10] Takahara J, Yamagishi S, Taki H, Morimoto A and Kobayashi T 1997 Guiding of a one dimensional optical beam with nanometer diameter Opt. Lett. 22475

[11] Babadjanyan A J, Margaryan N L and Nerkararyan Kh V 2000 Superfocusing of surface polaritons in the conical structures J. Appl. Phys. 873785

[12] Stockman M I 2004 Nanofocusing of optical energy in tapered plasmonic waveguides Phys. Rev. Lett. 93137404

[13] Vogel M W and Gramotnev D K 2007 Adiabatic nano-focusing of plasmons by metallic tapered rods in the presence of dissipation Phys. Lett. A 363507

[14] Kurihara K, Otomo A, Syouji A, Takahara J, Suzuki K and Yokoyama S 2007 Superfocusing modes of surface plasmon polaritons in conical geometry based on the quasi-separation of variables approach J. Phys. A 4012479

[15] Nerkararyan Kh V 1997 Superfocusing of a surface polariton in a wedge-like 
structure Phys. Lett. A 237103

[16] Gramotnev D K 2005 Adiabatic nanofocusing of plasmons by sharp metallic grooves: Geometrical optics approach J. Appl. Phys. 98104302

[17] Gramotnev D K and Vernon K C 2007 Adiabatic nanofocusing of plasmons by sharp metallic wedges Appl. Phys. B 867

[18] Gramotnev D K, Pile D F P, Vogel M W and Zhang X 2007 Local electric field enhancement during nanofocusing of plasmons by a tapered gap Phys. Rev. B 75 035431

[19] Kurihara K, Yamamoto K, Takahara J and Otomo A 2008 Superfocusing modes of surface plasmon polaritons in a wedge-shaped geometry obtained by quasi-separation of variables J. Phys. A 41295401

[20] Janunts N A, Bagdasaryan K S and Nerkararyan 2000 Superfocusing of surface polaritons in the system of two touching metallic semicylinders Phys. Lett. A 269257

[21] Lalayan A A, Bagdasaryan K S, Petrosyan P G and Nerkararyan Kh V 2002 Anomalous field enhancement from the superfocusing of surface plasmons at contacting silver surfaces J. Appl. Phys. 912965

[22] Nerkararyan Kh V, Hakhoumian A A and Babayan A E 2008 Terahertz surface plasmon-polariton superfocusing in coaxial cone semiconductor structures Plasmonics 3 27

[23] Janunts N A, Baghdasaryan K S, Nerkararyan Kh V and Hecht B 2005 Excitation and superfocusing of surface plasmon polaritons on a silver-coated optical fiber tip Opt. Commun. 253118

[24] Ruppin R 2005 Effect of non-locality on nanofocusing of surface plasmon field intensity in a conical tip Phys. Lett. A 340299

[25] Babayan A E and Nerkararyan Kh V 2007 The strong localization of surface plasmon polariton on a metal-coated tip of optical fiber Ultramicroscopy 1071136

[26] Vernon K C, Gramotnev and Pile D F P 2007 Adiabatic nanofocusing of plasmons by a sharp metal wedge on a dielectric substrate J. Appl. Phys. 101104312

[27] Ding W, Andrews S R and Maier S A 2007 Internal excitation and superfocusing of surface plasmon polaritons on a silver-coated optical fiber tip Phys. Rev. A 75 063822

[28] Bouhelier A, Renger J, Beversluis M R and Novotny L 2003 Plasmon-coupled tip-enhanced near-field optical microscopy J. Microsc. 210220 
[29] Maier S A, Andrews S R, Martín-Moreno L and Gracía-Vidal 2006 Terahertz surface plasmon-polariton propagation and focusing on periodically corrugated metal wires Phys. Rev. Lett. 97176805

[30] Pile D F P and Gramotnev D K 2006 Adiabatic and nonadiabatic nanofocusing of plasmons by tapered gap plasmon waveguides Appl. Phys. Lett. 89 041111, 119901 (erratum)

[31] Takahara J and Kusunoki F 2007 Guiding and nanofocusing of two-dimensional optical beam for nanooptical integrated circuits IEICETrans. Electron. E90C 87

[32] Issa N A and Guckenberger 2007 Optical nanofocusing on tapered metallic waveguides Plasmonics 231

[33] Gramotnev D K, Vogel M W and Stockman M I 2008 Optimized nonadiabatic nanofocusing of plasmons by tapered metal rods J. Appl. Phys. 104034311

[34] Tanaka K, Burr G W, Maletzky and Fischer 2008 Superfocussing in a metal-coated tetrahedral tip by dimensional reduction of surface-to edge-plasmon modes Appl. Phys. B 93257

[35] Verhagen E, Polman A and Kuipers L 2008 Nanofocusing in laterally tapered plasmonic waveguides Opt. Express 1645

[36] Ropers C, Neacsu C C, Elsaesser T, Albrecht M, Raschke M B and Lienau C 2007 Grating-coupling of surface plasmons onto metallic tips: a nanoconfined light source Nano Lett. 72784

[37] Ropers C, Neacsu C C, Raschke M B, Albrecht M, Lienau C and Elsaesser T 2008 Light confinement at ultrasharp metallic tips Jpn. J. Appl. Phys. 476051

[38] Chang D E, Sørensen A S, Hemmer P R and Lukin M D 2006 Quantum optics with surface plasmons Phys. Rev. Lett. 97053002

[39] Chang D E, Sørensen A S, Hemmer P R and Lukin M D 2007 Strong coupling of single emitters to surface plasmons Phys. Rev. B 76035420

[40] Micic M, Klymyshyn N, Suh Y D and Lu H P 2003 Finite element method simulation of the field distribution for AFM tip-enhanced surface-enhanced Raman scanning microscopy J. Phys. Chem. B 1071574

[41] Demming A L, Festy F and Richards D 2005 Plasmon resonances on metal tip: Understanding tip-enhanced Raman scattering J. Chem. Phys. 122184716

[42] Sánchez E J, Novotony L and Xie X S 1999 Near-field fluorescence microscopy based on two-photon excitation with metal tips Phys. Rev. Lett. 824014 
[43] Passian A, Wig A, Meriaudeau F and Ferrell T L 2002 Potential distribution and field intensity for a hyperboloidal probe in a uniform field J. Vac. Sci. Technol. B 2076 [44] Passian A, Ritchie R H, Lereu A L, Thundat T and Ferrell T L 2005 Curvature effects in surface plasmon dispersion and coupling Phys. Rev. B 71115425

[45] Lebedev N N 1972 Special Functions and their Applications (New York: Dover)

[46] Buchholz H 1969 The confluent Hypergeometric Function with Special Emphasis on its Applications (Berlin: Springer)

[47] Sommerfeld A 1949 Partial Differential Equation in Physics (New York: Academic Press Inc.)

[48] Samble J R, Bradbery G W and Yang F 1991 Optical excitation of surface plasmons: an introduction Contemp. Phys. 32173

[49] Kurihara K, Nakamura K and Suzuki K 2002 Asymmetric SPR sensor response curve-fitting equation for the accurate determination of SPR resonance angle Sens. Actuator B 8649

[50] Farlow S J 1993 Partial Differential Equations for Scientists and Engineers (New York: Dover)

[51] Jackson J D 1998 Classical electrodynamics 3rd edn (Hoboken: Wiley)

[52] Temme N M 1996 Special Function: An Introduction to the Classsical Functions of Mathematical Physics (New York: Wiley)

[53] Abramowitz M and Stegun I A 1972 Handbook of Mathematical Functions with Formulas, Graphs, and Mathematical Tables (New York: Dover)

[54] Slater L J 1960 Confluent Hypergeometric Functions (Cambridge: Cambridge University Press)

[55] Olver F W J 1997 Asymptotics and Special Functions (Natick: A K Peters)

[56] Dunster T M 1989 Uniform asymptotic expansions for Whittaker's confluent hypergeometric functions SIAM J. Math. Anal. 20744

[57] Innes R A and Sambles J R 1987 Optical characterization of gold using surface plasmon-polaritons J. Phys. F: Met. Phys. 17277

[58] Polyanin A D and Manzhirov 2007 Handbook of Mathematics for Engineers and Scientists (Boca Raton: Chapman and Hall/CRC Press)

[59] Arfken G B and Weber H J 2005 Mathematical Methods for Physicists $6^{\text {th }}$ edn (Amsterdam: Elsevier)

[60] Messiah A 1958 Quantum Mechanics (New York: Dover) 
[61] Dzieciol A, Yngve S and Fröman P O 1999 Coulomb wave functions with complex values of the variable and the parameters J. Math. Phys. 406145

[62] Seaton M J 2002 Coulomb functions for attractive and repulsive potentials and for positive and negative energies Comput. Phys. Comm. 146225

[63] Michel N 2007 Precise Coulomb wave functions for a wide range of complex $\ell$, $\eta$ and z Comput. Phys. Comm. 176232 


\section{Figure Captions}

\section{Figure 1.}

Geometry of a circular paraboloidal structure for the metallic solid paraboloid and the metallic hollow paraboloid. $\rho_{0}$ is the radius of curvature for the circular paraboloidal structure at the apex. $\varepsilon_{1}$ and $\varepsilon_{2}$ are the permittivities inside and outside the paraboloid, respectively. $H_{\phi}(\xi, \eta)$ is the magnetic field for plasmonic modes at paraboloidal coordinates $(\xi, \eta, \phi)$, which is directed along the $\phi$-axis and independent of the azimuthal angle $\phi$ because of the azimuthal symmetry. $\eta=\eta_{0}$ is the equation for the surface of the circular paraboloidal structure. $r$ is the distance from the origin to the point for the magnetic field of interest.

\section{Figure 2.}

Curves of constant $\xi$ and $\eta$ for paraboloidal coordinates in the cross-section of the y-z plane, calculated by (1). The curves of constant $\xi$ and $\eta$ are indicated by broken and solid lines, respectively.

\section{Figure 3.}

Numerical calculations of the zeroth-order unified separation quantity at $\xi=0$, $\zeta_{u}^{(0)}(0)$, as a function of the parameter $\eta_{0}$ for the paraboloidal surface $\eta=\eta_{0}$ when $\xi=0, \varepsilon_{m}=-20, \varepsilon_{d}=1$, and $s k_{0}=2 \pi$ in (110). The specific value of $\eta_{0}$ for $\zeta_{u}^{(0)}(0)=0$ is obtained as $\eta_{0}=0.429976$ from (116).

\section{Figure 4 .}

Numerical calculations of the zeroth-order unified separation quantity $\zeta_{u}^{(0)}(\xi)$ as a function of $\xi$ for various value of $\eta_{0}\left(=\rho_{0} / \lambda_{0}\right)$ when $\varepsilon_{m}=-20, \varepsilon_{d}=1$, and $s k_{0}=2 \pi$ in (110). For convenience of explanation, $\zeta_{u}^{(0)}(\xi)-\zeta_{u}^{(0)}(0)$ is indicated with a solid line. The broken lines are fitting curves based upon (119).

\section{Figure 5.}

Numerical calculations of the ratio $k_{m p}(\xi) / k_{m p}(0)$ in (128) as a function of $\xi$ for various values of $\eta_{0}\left(=\rho_{0} / \lambda_{0}\right)$ obtained by using $\varepsilon_{m}=-20, \varepsilon_{d}=1$, and specific 
values of $p_{1}, p_{2}$ and $p_{3}$ in table 1 .

\section{Figure 6.}

Electric field lines of the zeroth-order plasmonic mode of the metallic hollow paraboloid for (a) $\eta_{0}\left(=\rho_{0} / \lambda_{0}\right)=0.3$ and (b) 0.6 , obtained using (199) at $t=\pi / 2 \omega$. In the calculations of the unified radial and the extended angular functions in (199), $\varepsilon_{1}=1$ and $\varepsilon_{2}=-20$ are used. The blue and red lines indicate the clockwise and counterclockwise loops, respectively, of the line of electric force. The geometrical dimensions of the horizontal and vertical axes are normalized by the wavelength in vacuum $\lambda_{0}$.

\section{Figure 7.}

Electric field lines of the zeroth-order plasmonic mode of the metallic hollow paraboloid for $\eta_{0}\left(=\rho_{0} / \lambda_{0}\right)=0.429976$, the specific value of $\eta_{0}$ for $\zeta_{u}^{(0)}(0)=0$ when $\varepsilon_{1}=1$ and $\varepsilon_{2}=-20$. The other calculation conditions are the same as those in figure 6 . The blue and red lines indicate the clockwise and counterclockwise loops, respectively, of the line of electric force. The geometrical dimensions of the horizontal and vertical axes are normalized by the wavelength in vacuum $\lambda_{0}$. 


\section{Table Captions}

\section{Table 1.}

Values of the parameters in (119) for the specific value of $\eta_{0}\left(=\rho_{0} / \lambda_{0}\right)$. The values of $\zeta_{\mathrm{u}}^{(0)}(0)$ are numerically calculated in the same manner as that employed for drawing the solid line in figure 3 . The fitting parameters of $p_{1}, p_{2}$, and $p_{3}$ are obtained by fitting the curves in figure 4 . The values of $p_{1}\left(p_{2}+p_{3}\right)$ equal to $\zeta_{u}^{\prime(0)}(0)$ in (120) are simply calculated from the fitting parameters just obtained. 


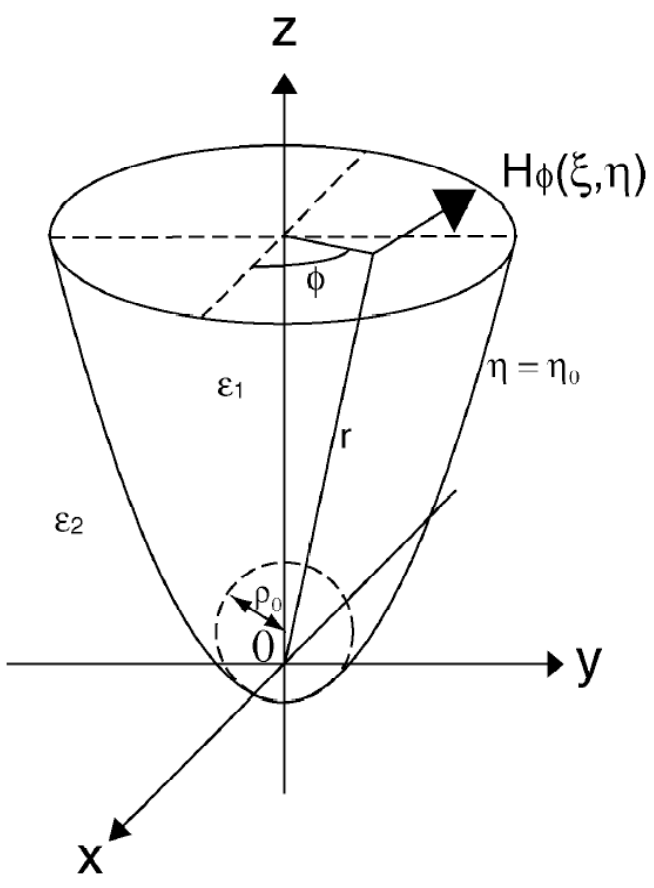

Fig. 1 


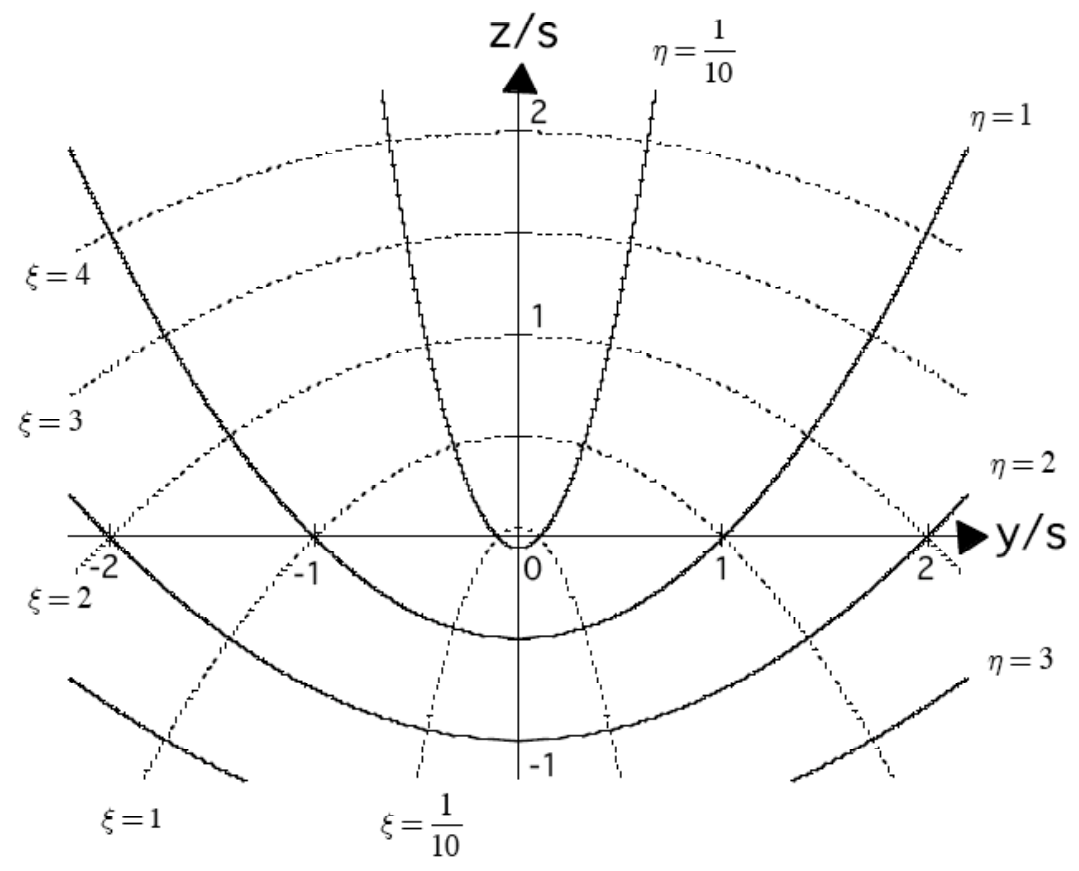

Fig.2 


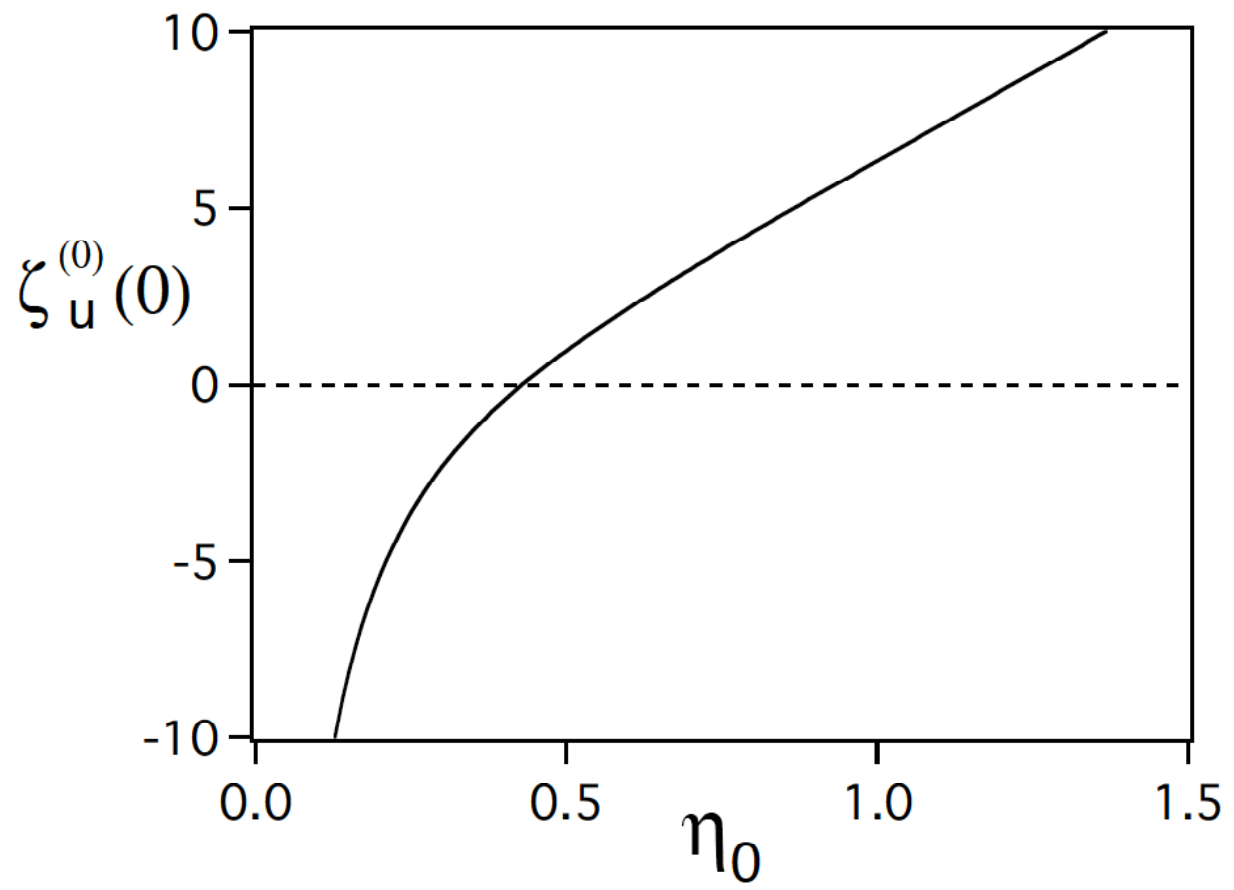

Fig.3 


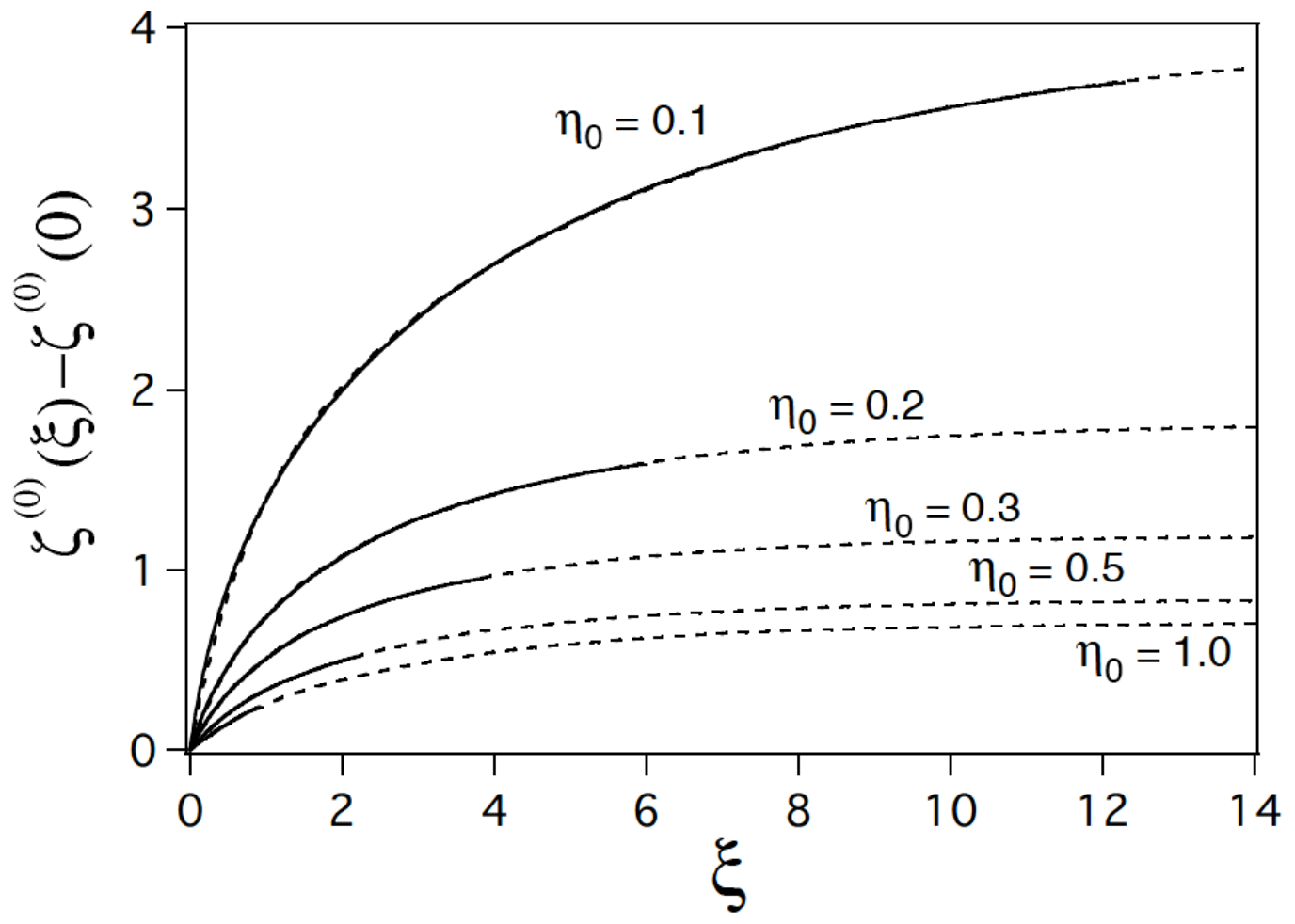

Fig.4 


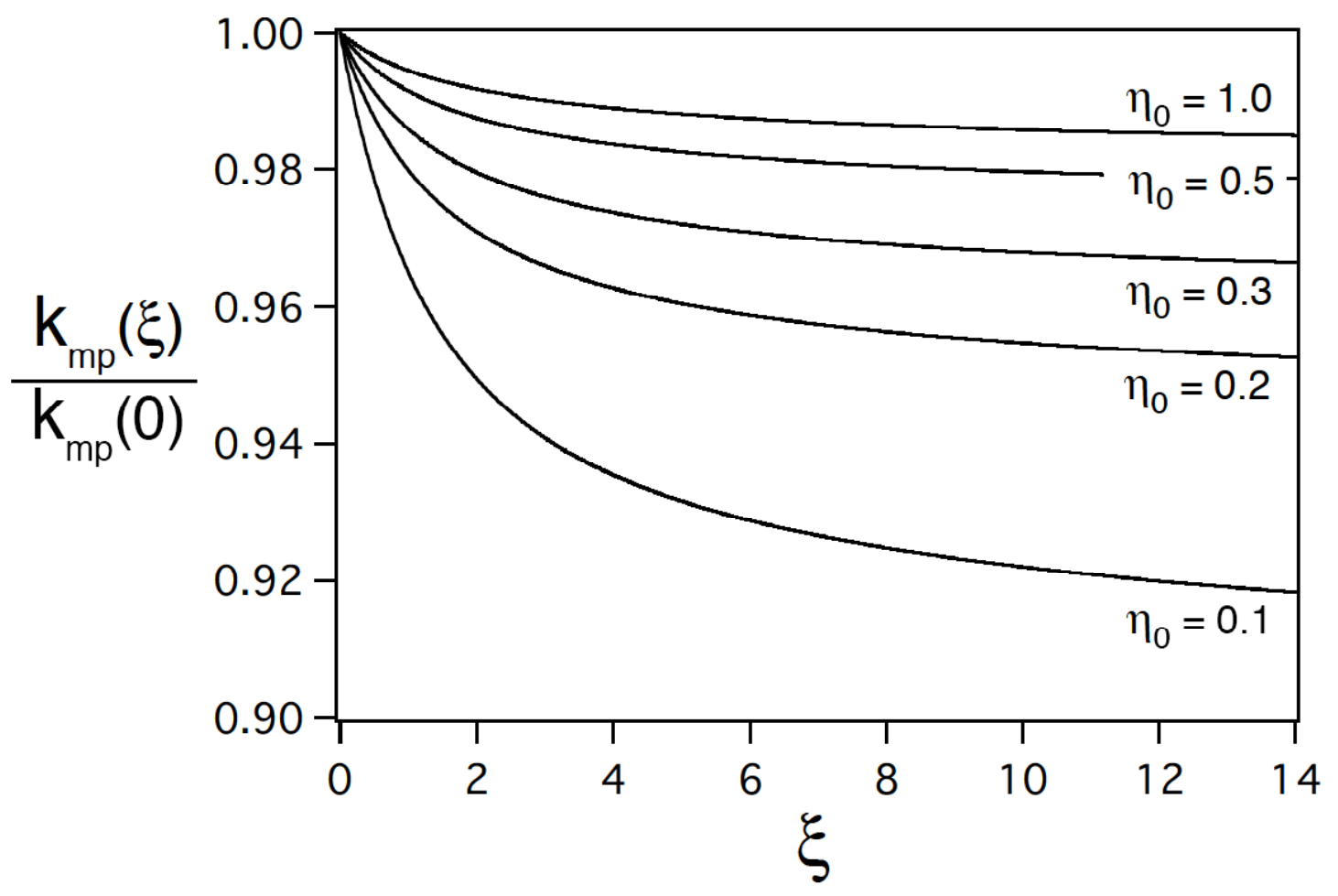

Fig.5 
(a)

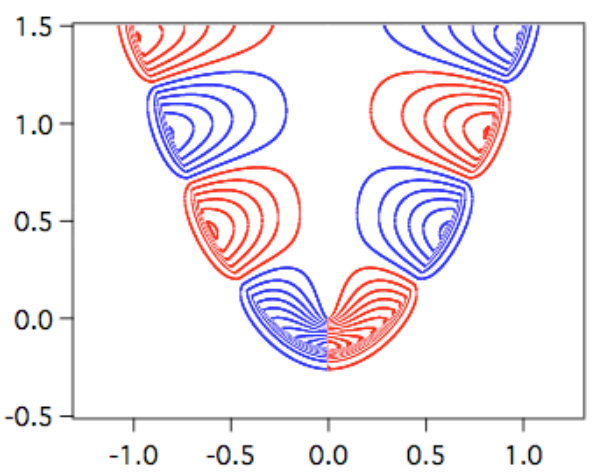

(b)

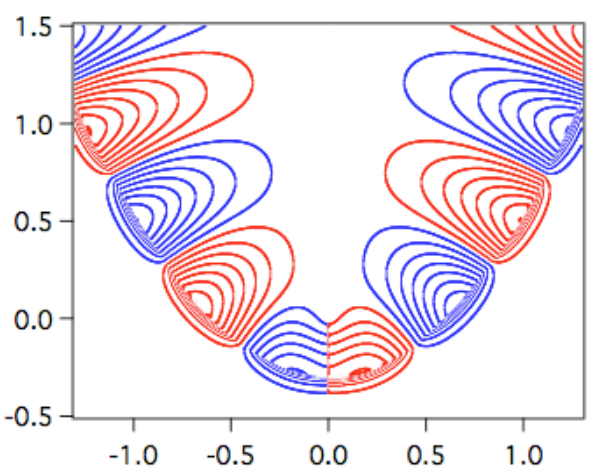

Fig.6 


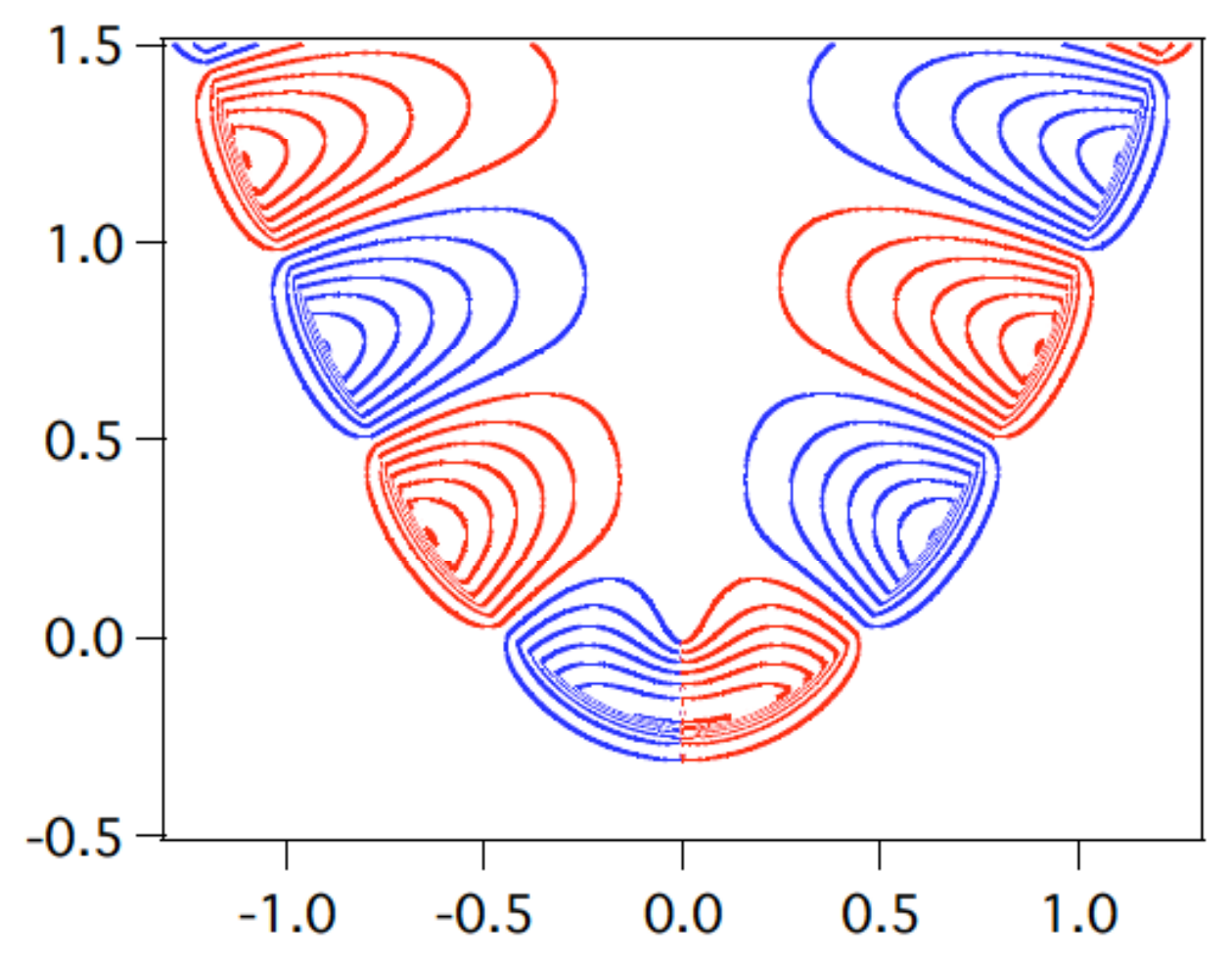

Fig.7 


\begin{tabular}{rrrrrr}
$\eta_{0}$ & $\zeta_{u}^{(0)}(0)$ & $p_{1}$ & $p_{2}$ & $p_{3}$ & $p_{1}\left(p_{2}+p_{3}\right)$ \\
\hline 0.1 & -13.39 & 3.960 & 0.3769 & 0.1921 & 2.253 \\
0.2 & -5.39 & 1.811 & 0.3736 & 0.2895 & 1.201 \\
0.3 & -2.32 & 1.186 & 0.3604 & 0.3329 & 0.8225 \\
0.4 & -0.46 & 0.9347 & 0.3336 & 0.3394 & 0.6291 \\
0.429976 & 0 & 0.9034 & 0.3269 & 0.3263 & 0.5901 \\
0.5 & 0.96 & 0.8336 & 0.2975 & 0.3245 & 0.5185 \\
0.6 & 2.17 & 0.8061 & 0.2574 & 0.3031 & 0.4518 \\
0.7 & 3.27 & 0.7832 & 0.2147 & 0.3088 & 0.4100 \\
0.8 & 4.31 & 0.7515 & 0.2147 & 0.3088 & 0.3934 \\
0.9 & 5.33 & 0.7283 & 0.2147 & 0.3088 & 0.3813 \\
1.0 & 6.34 & 0.7087 & 0.2147 & 0.3088 & 0.3710 \\
\hline
\end{tabular}

Table 1 